\title{
Equivariant Lefschetz maps for simplicial complexes and smooth manifolds
}

\author{
Heath Emerson • Ralf Meyer
}

Received: 30 May 2008 / Revised: 8 December 2008 / Published online: 21 April 2009

(C) The Author(s) 2009. This article is published with open access at Springerlink.com

\begin{abstract}
Let $X$ be a locally compact space with a continuous proper action of a locally compact group $G$. Assuming that $X$ satisfies a certain kind of duality in equivariant bivariant Kasparov theory, we can enrich the classical construction of Lefschetz numbers for self-maps to an equivariant K-homology class. We compute the Lefschetz invariants for self-maps of finite-dimensional simplicial complexes and smooth manifolds. The resulting invariants are independent of the extra structure used to compute them. Since smooth manifolds can be triangulated, we get two formulas for the same Lefschetz invariant in this case. The resulting identity is closely related to the equivariant Lefschetz Fixed Point Theorem of Lück and Rosenberg.
\end{abstract}

Mathematics Subject Classification (2000) $\quad$ 19K35 · 46L80

\section{Introduction}

Euler characteristics and Lefschetz numbers of self-maps are important objects in algebraic topology. They can be refined for spaces with a group action. A purely topological approach to such equivariant Lefschetz invariants has been developed by Lück and Weber $[10,12]$. This article grew out of the authors' previous work on equivariant Euler characteristics in [5]. The applications in [5] dictated studying Euler

\footnotetext{
H. Emerson

Department of Mathematics and Statistics, University of Victoria, PO Box 3045 STN CSC, Victoria, BC V8W 3P4, Canada e-mail: hemerson@math.uvic.ca

R. Meyer $(\varangle)$

Mathematisches Institut and Courant Centre "Higher Order Structures",

Georg-August Universität Göttingen, Bunsenstraße 3-5, 37073 Göttingen, Germany

e-mail: rameyer@uni-math.gwdg.de
} 
characteristics in the setting of bivariant Kasparov theory. Here we extend the framework of [5] to produce Lefschetz invariants as well.

The main ingredient in our definition of the Lefschetz invariant is a certain duality in bivariant Kasparov theory. Let $X$ be a locally compact space and let $G$ be a locally compact group acting on $X$. A $G$-equivariant abstract dual for $X$ is a pair $(\mathscr{P}, \Theta)$ consisting of a $G$-C*-algebra $\mathscr{P}$ and a class $\Theta \in \operatorname{RKK}_{n}^{G}(X ; \mathbb{C}, \mathscr{P})$ for some $n \in \mathbb{N}$ such that Kasparov product with $\Theta$ induces isomorphisms

$$
\mathrm{KK}_{*}^{G}(\mathscr{P} \otimes A, B) \cong \mathrm{RKK}_{*+n}^{G}(X ; A, B)
$$

for all $G$-C*-algebras $A$ and $B$; Kasparov [8] defines the groups $\mathrm{KK}_{*}^{G}(\mathscr{P} \otimes A, B)$ and $\mathrm{RKK}_{*+n}^{G}(X ; A, B)$ appearing here.

Given an abstract dual for $X$, we define an equivariant Euler characteristic of $X$ in $\mathrm{KK}_{0}^{G}\left(\mathscr{C}_{0}(X), \mathbb{C}\right)$ and an equivariant Lefschetz map

$$
\text { Lef : } \operatorname{RKK}_{*}^{G}\left(X ; \mathscr{C}_{0}(X), \mathbb{C}\right) \rightarrow \mathrm{KK}_{*}^{G}\left(\mathscr{C}_{0}(X), \mathbb{C}\right)
$$

The construction of these maps is explained in the body of the paper, see also [5]. The equivariant Euler characteristic is already studied in [5], the Lefschetz map is studied in [6], even in the more general setting of groupoid actions, which includes relative Euler characteristics and Lefschetz maps for bundles of spaces. Here we only consider the simpler case of a single space with a group action.

In order to actually compute the invariants, it is useful to have a bit more structure, which is formalised in the notion of a Kasparov dual in [5,6].

If $X$ is compact, then

$$
\operatorname{RKK}_{*}^{G}\left(X ; \mathscr{C}_{0}(X), \mathbb{C}\right) \cong \mathrm{KK}_{*}^{G}(\mathscr{C}(X), \mathscr{C}(X))
$$

because both groups are defined by the same cycles. Hence the domain of the equivariant Lefschetz map consists of morphisms from $X$ to $X$ in an appropriate category in this case. In general, there is a canonical map

$$
\mathrm{KK}_{*}^{G}\left(\mathscr{C}_{0}(X), \mathscr{C}_{0}(X)\right) \rightarrow \mathrm{RKK}_{*}^{G}\left(X ; \mathscr{C}_{0}(X), \mathbb{C}\right)
$$

(see [6, Sect. 4.1.4]). Roughly speaking, the group on the right hand side also contains non-proper self-maps of $\mathscr{C}_{0}(X)$. In particular, any $G$-equivariant continuous map $\psi: X \rightarrow X$ yields a class in $\operatorname{RKK}_{0}^{G}\left(X ; \mathscr{C}_{0}(X), \mathbb{C}\right)$, to which we can apply the Lefschetz map to get $\operatorname{Lef}(\psi) \in \mathrm{KK}_{0}^{G}\left(\mathscr{C}_{0}(X), \mathbb{C}\right)$.

We are going to compute Lef $(\psi)$ in two important cases where a Kasparov dual is available - for simplicial complexes and smooth manifolds - and compare the results. An important point is that Lef $(\psi)$ does not depend on the dual that we use to compute it. Since any smooth manifold can be triangulated, we therefore get two formulas for Lef $(\psi)$. The equality of these two formulas amounts to a Lefschetz Fixed Point Theorem in $\mathrm{KK}^{G}$. The result is comparable to the Equivariant Lefschetz Fixed Point Theorem of Lück and Rosenberg in [10]. However, there are some differences. On 
the one hand, their invariant is finer than ours. On the other hand, we are able to weaken the standard transversality assumption on $\psi$ to deal with the case where the fixed point set of $\psi$ has strictly positive dimension. Instead of orientation data at a fixed point, we get a contribution of the Euler characteristic of the fixed point set. In the transverse case, we describe the local orientation data in a different way than Lück and Rosenberg. The local data for us is an element of the representation ring of the isotropy subgroup of the fixed point. It can be described using Clifford algebras and their representation theory. Finally, our methods allow the group $G$ to be non-discrete.

Lefschetz invariants exist for general elements of $\mathrm{KK}_{*}^{G}\left(\mathscr{C}_{0}(X), \mathscr{C}_{0}(X)\right)$, not just for maps. We will address this kind of generalisation in a future article. As in the Euler characteristic computations in [5], in the analysis presented here, Lef $(\psi)$ appears as a linear combination of point evaluation classes, at least in the simplicial case. In this sense, the Lefschetz invariant of a map is always a 0 -dimensional object. This changes when we apply the Lefschetz map to more general elements in $\operatorname{RKK}_{*}^{G}\left(X ; \mathscr{C}_{0}(X), \mathbb{C}\right)$ : on that domain, the Lefschetz map is split surjective by [6, Proposition 4.26], so that the Lefschetz map yields arbitrarily complicated K-homology classes. Of course, this also makes computations more difficult. In another forthcoming article, we shall compute Lefschetz invariants of geometric cycles or correspondences as defined by Baum and his coauthors ([1,2]) and by Connes and Skandalis [3]. But these computations use a different Kasparov dual in order to express everything in terms of geometric cycles. The computations here are closer to those in [5].

There are some similarities between the results here and the Lefschetz Fixed Point Formula in [4], which is also based on the method of Poincaré duality. However, each deal with different situations: in [4] the trace of certain automorphisms of the crossed product $\mathscr{C}_{0}(X) \ltimes G$ on $\mathrm{K}_{*}\left(\mathscr{C}_{0}(X) \ltimes G\right)$ is computed. Our approach here only deals with special automorphisms that act identically on $G$; but it yields a refined invariant in $\mathrm{KK}_{0}^{G}\left(\mathscr{C}_{0}(X), \mathbb{C}\right)$ instead of just a number.

The contents of this article is as follows. In Sect. 2, we briefly recall the notion of a Kasparov dual and define the Lefschetz map. Section 3 contains our three main results. Theorem 1 provides a formula for $\operatorname{Lef}(\psi)$ in the simplicial case and Theorem 2 in the smooth case; Theorem 3 combines them into a Lefschetz Fixed Point Theorem. The proofs appear in Sect. 4 for the simplicial case and in Sect. 5 for the smooth case.

\section{Kasparov duality and the Lefschetz map}

The general framework of duals and Lefschetz maps is explained carefully in [6]. Here we only recall the definition of a Kasparov dual for a $G$-space $X$, where $X$ is a locally compact space and $G$ a locally compact group. As in [6], we write $\mathbb{1}=\mathbb{C}$. If we worked with real $C^{*}$-algebras, we would use $\mathbb{R}$ instead.

A Kasparov dual for $X$ consists of an $X \rtimes G$ - $C^{*}$-algebra $\mathscr{P}$ and two classes

$$
\Theta \in \operatorname{RKK}_{n}^{G}(X ; \mathbb{1}, \mathscr{P}), \quad D \in \mathrm{KK}_{-n}^{G}(\mathscr{P}, \mathbb{1}),
$$


satisfying some conditions (see [6, Definition 4.1]). These ensure that the maps

$$
\begin{aligned}
& \mathrm{PD}: \mathrm{KK}_{i-n}^{G}(A \otimes \mathscr{P}, B) \rightarrow \mathrm{RKK}_{i}^{G}(X ; A, B), \quad f \mapsto \Theta \otimes \mathscr{P} f, \\
& \mathrm{PD}^{*}: \mathrm{RKK}_{i}^{G}(X ; A, B) \rightarrow \mathrm{KK}_{i-n}^{G}(A \otimes \mathscr{P}, B), \quad g \mapsto(-1)^{n i} T_{\mathscr{P}}(g) \otimes \mathscr{P} D,
\end{aligned}
$$

are inverse to each other. Here the map $T_{\mathscr{P}}$ combines three maps

$$
\begin{aligned}
\operatorname{RKK}_{*}^{G}(X ; A, B) & =\mathscr{R} \mathrm{KK}_{*}^{G}\left(X ; \mathscr{C}_{0}(X, A), \mathscr{C}_{0}(X, B)\right) \\
& \rightarrow \mathscr{R} \mathrm{KK}_{*}^{G}\left(X \times X ; \mathscr{C}_{0}(X, A) \otimes \mathscr{P}, \mathscr{C}_{0}(X, B) \otimes \mathscr{P}\right) \\
& \rightarrow \mathscr{R} \mathrm{KK}_{*}^{G}(X ; A \otimes \mathscr{P}, B \otimes \mathscr{P}) \rightarrow \mathrm{KK}_{*}^{G}(A \otimes \mathscr{P}, B \otimes \mathscr{P})
\end{aligned}
$$

the first map is the exterior product, the second one is induced by the diagonal embed$\operatorname{ding} X \rightarrow X \times X$, and the third one is the forgetful map. In our computations, we will only use the following special case:

Lemma 1 Let $\psi: X \rightarrow X$ be a G-equivariant map. Let $\psi^{*}\left(\Delta_{X}\right)$ be the class in $\operatorname{RKK}_{0}^{G}\left(X ; \mathscr{C}_{0}(X), \mathbb{1}\right)$ of the *-homomorphism induced by the map $X \rightarrow X \times X$, $x \mapsto(x, \psi(x))$, as in [6, Example 4.19]. Then

$$
T_{\mathscr{P}}\left(\psi^{*} \Delta_{X}\right) \in \mathrm{KK}_{0}^{G}\left(\mathscr{C}_{0}(X) \otimes \mathscr{P}, \mathscr{P}\right)
$$

is represented by the G-equivariant *-homomorphism

$$
\mu_{\psi}: \mathscr{C}_{0}(X) \otimes \mathscr{P} \rightarrow \mathscr{P}, \quad f \otimes a \mapsto(f \circ \psi) \cdot a,
$$

where the multiplication uses the $X$-structure on $\mathscr{P}$.

Proof The proof is straightforward.

The Lefschetz map for $X$ is the composition

$$
\begin{aligned}
& \text { Lef : } \operatorname{RKK}_{*}^{G}\left(X ; \mathscr{C}_{0}(X), \mathbb{1}\right) \stackrel{\mathrm{PD}^{*}}{\longrightarrow} \mathrm{KK}_{*+n}^{G}\left(\mathscr{C}_{0}(X) \otimes \mathscr{P}, \mathbb{1}\right) \\
& \stackrel{\bar{\Theta} \otimes \mathscr{C}_{0}(X) \otimes \mathscr{P} \sqcup}{\longrightarrow} \mathrm{KK}_{*}^{G}\left(\mathscr{C}_{0}(X), \mathbb{1}\right) .
\end{aligned}
$$

Here $\bar{\Theta}$ is obtained from $\Theta$ by applying the forgetful map

$$
\operatorname{RKK}_{n}^{G}(X ; \mathbb{1}, \mathscr{P}) \rightarrow \mathrm{KK}_{n}^{G}\left(\mathscr{C}_{0}(X), \mathscr{C}_{0}(X) \otimes \mathscr{P}\right)
$$

Given a $G$-equivariant map $f: X \rightarrow X$, we define

$$
\operatorname{Lef}(\psi):=\operatorname{Lef}\left(\psi^{*} \Delta_{X}\right)
$$


where we use $\psi^{*} \Delta_{X} \in \operatorname{RKK}_{0}^{G}\left(X ; \mathscr{C}_{0}(X), \mathbb{1}\right)$ as defined in Lemma 1. Plugging in (3) and Lemma 1 , this becomes

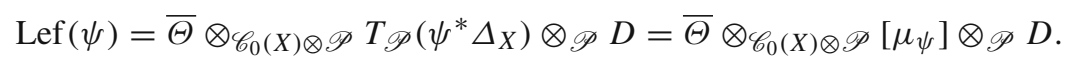

Since the class of $\psi^{*} \Delta_{X}$ in $\operatorname{RKK}_{0}^{G}\left(X ; \mathscr{C}_{0}(X), \mathbb{1}\right)$ depends only on the equivariant homotopy class of $\psi$, so does $\operatorname{Lef}(\psi)$. The Lefschetz invariant of the identity map is the equivariant Euler characteristic of $X$ :

$$
\operatorname{Eul}_{X}:=\operatorname{Lef}\left(\operatorname{id}_{X}\right) \in \mathrm{KK}_{0}^{G}\left(\mathscr{C}_{0}(X), \mathbb{1}\right)
$$

This agrees with the definition in [5].

All choices of a Kasparov dual $(\mathscr{P}, \Theta, D)$ yield the same Lefschetz map. This is shown in [5] for the equivariant Euler characteristic; as observed in [6], the proof generalises to the Lefschetz map. We will use this fact when $X$ is, at the same time, a smooth Riemannian manifold with $G$ acting isometrically and a simplicial complex with $G$ acting simplicially. Both the smooth and the combinatorial structure provide Kasparov duals for $X$. These two duals yield different formulas for $\operatorname{Lef}(\psi)$. But they must produce the same class in $\mathrm{KK}_{0}^{G}\left(\mathscr{C}_{0}(X), \mathbb{1}\right)$. The equality between both results is our equivariant Lefschetz formula.

\section{Statement of the main results}

We first consider the simplest possible case. We let $G$ be trivial and let $X$ be a connected compact manifold equipped with a triangulation. Let $C_{\bullet}(X)$ be the resulting simplicial chain complex. Let $\psi: X \rightarrow X$ be a self-map.

We can find a smooth map $\tilde{\psi}: X \rightarrow X$ that is homotopic to $\psi$ and whose graph is transverse to the diagonal. When we use the Kasparov dual from the smooth structure on $X$, we get

$$
\operatorname{Lef}(\psi)=\operatorname{Lef}(\tilde{\psi})=\sum_{p \in \operatorname{Fix}(\tilde{\psi})} \operatorname{sign} \operatorname{det}\left(\operatorname{id}_{T_{p} X}-D_{p} \tilde{\psi}\right) \cdot[\mathrm{ev}]
$$

where $D_{p} \tilde{\psi}$ denotes the derivative of $\tilde{\psi}$ at $p$ and $[\mathrm{ev}] \in \mathrm{K}_{0}(X)$ denotes the class of point evaluations. Since $X$ is connected and KK-theory is homotopy invariant, all point evaluations have the same K-homology class. The numerical factor in front of $[\mathrm{ev}]$ is the usual local formula for the Lefschetz number of $\tilde{\psi}$ in terms of fixed points.

The triangulation provides another Kasparov dual for $X$. This dual yields

$$
\operatorname{Lef}(\psi)=\sum_{d=0}^{\operatorname{dim} X}(-1)^{d} \operatorname{tr}\left(\Psi_{d}: C_{d}(X) \rightarrow C_{d}(X)\right) \cdot[\mathrm{ev}],
$$

where $\Psi_{\bullet}: C_{\bullet}(X) \rightarrow C_{\bullet}(X)$ is the chain map induced by $\psi$ (or a cellular approximation of $\psi)$. A familiar trick replaces the spaces of cycles $C_{d}(X)$ by the homology spaces 
$\mathrm{H}_{d}(X)$ in the last formula, expressing the Lefschetz number as a global homological invariant.

The equivariant generalisation of our Lefschetz formulas requires some preliminary notation. First we explain the formula for the combinatorial case, then for the smooth case.

\subsection{The combinatorial Lefschetz map}

Let $X$ be a finite-dimensional simplicial complex and let $G$ be a locally compact group acting smoothly and simplicially on $X$ (that is, stabilisers of points are open). The finite-dimensionality assumption is needed for the construction of a Kasparov dual in [5] to work. It can probably be dropped if we use a more sophisticated dual. Assume that $X$ admits a colouring (that is, $X$ is typed) and that $G$ preserves the colouring. This ensures that if $g \in G$ maps a simplex to itself, then it fixes that simplex pointwise.

Let $S X$ be the set of (non-degenerate) simplices of $X$ and let $S_{d} X \subseteq S X$ be the subset of $d$-dimensional simplices. The group $G$ acts smoothly on the discrete set $S X$ preserving the decomposition $S X=\bigsqcup S_{d} X$. Decompose $S X$ into $G$-orbits. For each orbit $\dot{\sigma} \subseteq S X$, choose a representative $\sigma \in S X$ and let $\xi_{\sigma} \in X$ be its barycentre and $\operatorname{Stab}(\sigma) \subseteq G$ its stabiliser. Restriction to the orbit of $\xi_{\sigma}$ defines a $G$-equivariant *-homomorphism

$$
\xi_{\dot{\sigma}}: \mathscr{C}_{0}(X) \rightarrow \mathscr{C}_{0}(G / \operatorname{Stab}(\sigma)) \rightarrow \mathbb{K}\left(\ell^{2}(G / \operatorname{Stab} \sigma)\right)
$$

where the second map is the representation by pointwise multiplication operators. We let $\left[\xi_{\dot{\sigma}}\right]$ be its class in $\mathrm{KK}_{0}^{G}\left(\mathscr{C}_{0}(X), \mathbb{1}\right)$.

Let $\psi: X \rightarrow X$ be a $G$-equivariant self-map of $X$. It is too restrictive to assume $\psi$ to be simplicial-simplicial approximation requires us to refine the triangulations on domain and target independently, so that we may need two different triangulations of $X$ for a self-map. What we can achieve is that $\psi$ becomes a cellular map with respect to the canonical $\mathrm{CW}$-complex structure on a simplicial complex.

More precisely, $\psi$ is $G$-equivariantly homotopic to a $G$-equivariant cellular map. Hence we may assume without loss of generality that $\psi$ is itself cellular, so that it induces a $G$-equivariant chain map

$$
\Psi: C_{\bullet}(X) \rightarrow C_{\bullet}(X)
$$

where $C_{\bullet}(X)$ is the cellular chain complex of $X$; this is nothing but the chain complex of oriented simplices of $X$. A basis for $C_{\bullet}(X)$ is given by the set of (un)oriented simplices, by arbitrarily choosing an orientation on each simplex. We may describe the chain map $\Psi$ by its matrix coefficients $\Psi_{\sigma \tau} \in \mathbb{Z}$ with respect to this basis; thus the subscripts are unoriented simplices. For example, if $\psi$ maps a simplex to itself and reverses its orientation, then $\Psi_{\sigma, \sigma}=-1$. Since $\psi$ is $G$-equivariant, $\Psi_{g(\sigma), g(\sigma)}=\Psi_{\sigma \sigma}$. So the following makes sense. 
Definition 1 For $\dot{\sigma} \in G \backslash S_{d} X$, let $\mathrm{n}(\Psi, \dot{\sigma}):=(-1)^{d} \Psi_{\sigma \sigma} \in \mathbb{Z}$ for any choice of representative $\sigma \in \dot{\sigma}$.

Theorem 1 Let $X$ be a finite-dimensional coloured simplicial complex and let $G$ be a locally compact group that acts smoothly and simplicially on $X$, preserving the colouring. Let $\psi: X \rightarrow X$ be a G-equivariant self-map. Define $\mathrm{n}(\Psi, \dot{\sigma}) \in \mathbb{Z}$ and $\left[\xi_{\dot{\sigma}}\right] \in \mathrm{KK}_{0}^{G}\left(\mathscr{C}_{0}(X), \mathbb{1}\right)$ for $\dot{\sigma} \in G \backslash S X$ as above. Then

$$
\operatorname{Lef}(\psi)=\sum_{\dot{\sigma} \in G \backslash S X} \mathrm{n}(\Psi, \dot{\sigma})\left[\xi_{\dot{\sigma}}\right]
$$

Theorem 1 will be proved in Sect. 4, where we also recall the Kasparov dual for $X$.

\subsection{The K-orientation of a vector bundle automorphism}

Here we prepare for the Lefschetz formula for self-maps of manifolds. We need an invariant for vector bundle automorphisms that generalises the sign of the determinant of a linear map $A: V \rightarrow V$ on a single vector space. This is a refinement of a construction which appears in [4] in connection with the equivariant index of twisted Schrödinger operators. If $G$ is a group of orthogonal transformations of $\mathbb{R}^{n}$ and if $A \in \mathrm{Gl}_{n}(\mathbb{R})$ commutes with $G$, then a refinement of the sign of the determinant of $A$ is the virtual group character

$$
\chi_{G, A}: G \rightarrow\{ \pm 1\}, \quad \chi_{G, A}(g):=\operatorname{sign} \operatorname{det}\left(\left.A\right|_{\text {Fix }(g)}\right)
$$

The virtual representation of $G$ corresponding to $\chi_{G, A}$ is the solution to an equivariant index problem on $\mathbb{R}^{n}$. Here we take a different approach to the same invariant, which permits a generalisation of it to equivariant bundles. In the case where $Y$ is a point, the equivalence between the picture in [4] and the one presented here follows from the fact that both give a solution to the same equivariant index problem.

A very similar invariant to ours appears in the work of Lück and Rosenberg, but the connection to index theory and to representation theory seems absent.

We consider the following situation. Let $Y$ be a locally compact space, let $G$ be a locally compact group (or groupoid) acting continuously on $Y$, and let $\pi: E \rightarrow Y$ be a $G$-equivariant Euclidean $\mathbb{R}$-vector bundle over $E$, that is, $E$ comes equipped with a $G$-invariant inner product on its fibres. Let $A: E \rightarrow E$ be a $G$-equivariant vector bundle automorphism, that is, a $G$-equivariant continuous map $E \rightarrow E$ over $Y$ that restricts to $\mathbb{R}$-vector space isomorphisms on the fibres of $E$. We are going to define a $G$-equivariant $\mathbb{Z} / 2$-graded real line bundle $\operatorname{sign}(A)$ over $Y$.

Since we work with complex K-theory most of the time, we are mainly interested in the complexification $\operatorname{sign}(A) \otimes_{\mathbb{R}} \mathbb{C}$. Nevertheless, it is worth noting that the line bundles we get are complexifications of real line bundles.

If $Y$ is a point, then $G$-equivariant $\mathbb{Z} / 2$-graded real vector bundles over $Y$ correspond to real orthogonal virtual representations of $G$. The initial data is thus a real orthogonal representation of $G$ on a Euclidean space $\mathbb{R}^{n}$ and an invertible linear map 
$A: \mathbb{R}^{n} \rightarrow \mathbb{R}^{n}$ commuting with $G$. The sign is a virtual one-dimensional representation of $G$ and hence corresponds to a pair $(\chi, \varepsilon)$, where $\varepsilon \in\{0,1\}$ is the parity of the line bundle and $\chi: G \rightarrow\{-1,+1\}$ is a real-valued character. The parity $\varepsilon$ turns out to be 0 if $A$ preserves orientation and 1 if $A$ reverses orientation (see Example 2). In this sense, our invariant refines the orientation of $A$. As it happens, if the parity is even, then $\chi=\chi_{G, A}$ as in (8), and if the parity is odd, then $\chi=-\chi_{G, A}$. In particular, evaluating $\chi_{G, A}$ at the identity of $G$ yields the parity.

Definition 2 Let Cliff $(E)$ be the bundle of Clifford algebras associated to $E$; its fibre $\operatorname{Cliff}(E)_{x}$ is the (real) Clifford algebra of $E_{x}$ with respect to the given inner product.

We can also form $\operatorname{Cliff}(E)$ if $E$ carries an indefinite bilinear form. If the index of the bilinear form on $E$ is divisible by 8, then the fibres of $\operatorname{Cliff}(E)$ are isomorphic to matrix algebras. In this case, a $G$-equivariant spinor bundle for $E$ is a $\mathbb{Z} / 2$-graded real vector bundle $S_{E}$ together with a grading preserving, $G$-equivariant *-algebra isomorphism $c: \operatorname{Cliff}(E) \rightarrow \operatorname{End}\left(S_{E}\right)$. This representation is determined uniquely by its restriction to $E \subseteq \operatorname{Cliff}(E)$, which is a $G$-equivariant map $c: E \rightarrow \operatorname{End}\left(S_{E}\right)$ such that $c(x)$ is odd and symmetric and satisfies $c(x)^{2}=\|x\|^{2}$ for all $x \in E$. We only use spinor bundles in this special case.

Recall that the spinor bundle is uniquely determined up to tensoring with a $G$-equivariant real line bundle $L:$ if $c_{t}: E \rightarrow S_{t}$ for $t=1,2$ are two $G$-equivariant spinor bundles for $E$, then we define a $G$-equivariant real line bundle $L$ over $Y$ by

$$
L:=\operatorname{Hom}_{\mathrm{Cliff}(E)}\left(S_{1}, S_{2}\right)
$$

and the evaluation isomorphism $S_{1} \otimes L \stackrel{\cong}{\rightarrow} S_{2}$ intertwines the representations $c_{1}$ and $c_{2}$ of $\operatorname{Cliff}(E)$.

Definition 3 Let $A: E \rightarrow E$ be a $G$-equivariant vector bundle automorphism and let $A=T \circ\left(A^{*} A\right)^{1 / 2}$ be its polar decomposition with an orthogonal vector bundle automorphism $T: E \rightarrow E$.

Let $F$ be another $G$-equivariant vector bundle over $Y$ with a non-degenerate bilinear form, such that the signature of $E \oplus F$ is divisible by 8, so that $\operatorname{Cliff}(E \oplus F)$ is a bundle of matrix algebras over $\mathbb{R}$ and $E \oplus F$ has a $G$-equivariant spinor bundle, that is, there exists a $G$-equivariant linear map $c: E \oplus F \rightarrow \operatorname{End}(S)$ that induces an isomorphism of graded *-algebras

$$
\operatorname{Cliff}(E \oplus F) \cong \operatorname{End}(S) .
$$

Then

$$
c^{\prime}: E \oplus F \rightarrow \operatorname{End}(S), \quad(\xi, \eta) \mapsto c(T(\xi), \eta)
$$

yields another $G$-equivariant spinor bundle for $E \oplus F$. We let

$$
\operatorname{sign}(A):=\operatorname{Hom}_{\mathrm{Cliff}(E \oplus F)}\left(\left(S, c^{\prime}\right),(S, c)\right) .
$$

This is a $G$-equivariant $\mathbb{Z} / 2$-graded real line bundle over $Y$. 
Example 1 For example, consider $A=$ id. Choose $F, S$ and $c$ as in the definition. Then the sign of the identity map is $\operatorname{Hom}_{\operatorname{Cliff}(E \oplus F)}((S, c),(S, c))$. Since the spinor representation is fibrewise irreducible, the only fibrewise endomorphisms of $S$ that commute with the Clifford action are multiples of the identity map. The identity map is grading-preserving and commutes with $G$. Hence sign(id) is the trivial, evenly graded line bundle over $Y$ equipped with the trivial $G$-action.

Formally, we may think of the sign construction as follows. The set of equivariant $\mathrm{K}$-orientations on $X$ is in a natural way a module over the Abelian group of real equivariant line bundles. The procedure given above of twisting with an equivariant map $f: X \rightarrow X$ is invariant under twisting with a real equivariant line bundle, that is, it commutes with the module structure. Hence it must itself be given by module product with some equivariant real line bundle. The latter is the sign of $f$.

Lemma 2 The $G$-equivariant $\mathbb{Z} / 2$-graded real line bundle sign $(A)$ is well-defined, that is, it depends neither on the bundle $F$ nor on the spinor bundle $S$ for $E \oplus F$. Furthermore, $\operatorname{sign}(A)$ is a homotopy invariant of $A$ and has the following additivity properties:

- $\operatorname{sign}\left(A_{1} \circ A_{2}\right) \cong \operatorname{sign}\left(A_{1}\right) \otimes \operatorname{sign}\left(A_{2}\right)$ for two equivariant automorphisms $A_{1}, A_{2}: E \rightrightarrows E$ of the same bundle;

- $\operatorname{sign}\left(A_{1} \oplus A_{2}\right) \cong \operatorname{sign}\left(A_{1}\right) \otimes \operatorname{sign}\left(A_{2}\right)$ for two equivariant vector bundle automorphisms $A_{1}: E_{1} \rightarrow E_{1}$ and $A_{2}: E_{2} \rightarrow E_{2}$.

Proof Since the spinor bundle for $E \oplus F$ is unique up to tensoring with some line bundle $L$, changing the spinor bundle replaces $\operatorname{sign}(A)$ by $L \otimes \operatorname{sign}(A) \otimes L^{*}$ for some line bundle, which is canonically isomorphic to $\operatorname{sign}(A)$. Homotopy invariance of $\operatorname{sign}(A)$ follows because homotopic line bundles are isomorphic, and this implies that it suffices to treat orthogonal transformations $A$.

Let $E_{1}$ and $E_{2}$ be two $G$-equivariant vector bundles with $G$-equivariant spinor bundles $\left(S_{1}, c_{1}\right)$ and $\left(S_{2}, c_{2}\right)$. Let $A_{1}: E_{1} \rightarrow E_{1}$ and $A_{2}: E_{2} \rightarrow E_{2}$ be two vector bundle automorphisms. Equip $E:=E_{1} \oplus E_{2}$ with the automorphism $A:=A_{1} \oplus A_{2}$ and the $G$-equivariant spinor bundle $S:=S_{1} \otimes S_{2}$ with the representation

$$
c:=\left(c_{1} \otimes 1\right) \oplus\left(1 \otimes c_{2}\right): E_{1} \oplus E_{2} \rightarrow \operatorname{End}\left(S_{1} \otimes S_{2}\right)
$$

The canonical map

$$
\begin{aligned}
\operatorname{Hom}_{\mathrm{Cliff}\left(E_{1}\right)}\left(\left(S_{1}, c_{1}\right),\left(S_{1}, c_{1} \circ A_{1}\right)\right) & \otimes \operatorname{Hom}_{\mathrm{Cliff}\left(E_{2}\right)}\left(\left(S_{2}, c_{2}\right),\left(S_{2}, c_{2} \circ A_{2}\right)\right) \\
\rightarrow & \operatorname{Hom}_{\mathrm{Cliff}(E)}((S, c),(S, c \circ A))
\end{aligned}
$$

is an isomorphism.

For $A_{2}=\mathrm{id}$, this shows that $\operatorname{sign}\left(A_{1}\right)$ remains unchanged if we stabilise by another vector bundle with a spin structure. Hence $\operatorname{sign}(A)$ does not depend on $F$. We also get the second additivity property; the first one is trivial.

Example 2 By $\mathbb{R}$ we will understand the one-dimensional real vector space with positive parity, and by $\mathbb{R}^{\mathrm{op}}$ the same vector space with odd parity. 
By the definitions, if $Y$ is a point and $G$ is trivial, then $\operatorname{sign}(A)$ is either $\mathbb{R}$ or $\mathbb{R}^{\text {op }}$. Since $\operatorname{sign}(A)$ is homotopy invariant, all orientation-preserving maps $A$ have $\operatorname{sign}(A)=\operatorname{sign}(\mathrm{id})=\mathbb{R}$. A routine computation (see the next example) shows that the sign of the orientation reversal automorphism $x \mapsto-x$ on $\mathbb{R}$ is $\mathbb{R}^{\text {op }}$. Since any orientation-reversing map $A$ is homotopic to the direct sum of $x \mapsto-x$ and $\mathrm{id}_{\mathbb{R}^{n-1}}$, it follows from Lemma 2 that $\operatorname{sign}(A)=\mathbb{R}$ for orientation-preserving $A$ and $\operatorname{sign}(A)=\mathbb{R}^{\text {op }}$ for orientation-reversing $A$, as claimed above.

Example 3 Consider $G=\mathbb{Z} / 2$. We use similar notation as above, but decorate $\mathbb{R}$ (or $\mathbb{R}^{\mathrm{op}}$ ) by a subscript which is a character, as appropriate.

Let $\tau: G \rightarrow\{1\}$ be the trivial character and let $\chi: G \rightarrow\{+1,-1\}$ be the nontrivial character. Consider $A: \mathbb{R}_{\chi} \rightarrow \mathbb{R}_{\chi}, t \mapsto-t$. Then $\operatorname{sign}(A) \cong \mathbb{R}_{\chi}^{\text {op }}$ carries a non-trivial representation.

To see this, let $F$ be $\mathbb{R}_{\chi}$ with negative definite metric. Thus the Clifford algebra of $\mathbb{R}_{\chi} \oplus \mathbb{R}_{\chi}$ is Cliff $_{1,1} \cong \mathbb{M}_{2 \times 2}(\mathbb{R})$. Explicitly, the map

$$
c(x, y)=\left(\begin{array}{cc}
0 & x-y \\
x+y & 0
\end{array}\right)
$$

induces the isomorphism. We equip $\mathbb{R}^{2}$ with the representation $\tau \oplus \chi$, so that $c$ is equivariant.

Twisting by $A$ yields another representation

$$
c^{\prime}(x, y):=c(-x, y)=S c(x, y) S^{-1} \quad \text { with } \quad S=S^{-1}=\left(\begin{array}{cc}
0 & 1 \\
-1 & 0
\end{array}\right) .
$$

Since $S$ reverses the grading and exchanges the representations $\tau$ and $\chi$, it induces an isomorphism $\left(\mathbb{R}_{\tau} \oplus \mathbb{R}_{\chi}^{\text {op }}\right) \otimes \mathbb{R}_{\chi}^{\text {op } \cong} \stackrel{\cong}{\rightarrow} \oplus \mathbb{R}_{\chi}^{\text {op }}$. Hence $\operatorname{sign}(A)=\mathbb{R}_{\chi}^{\text {op }}$.

This result can be computed using the picture (8) instead. The fixed point set of the identity element of $\mathbb{Z} / 2$ is all of $\mathbb{R}$. The fixed point set of the non-trivial generator of $\mathbb{Z} / 2$ is $\{0\} \subset \mathbb{R}$. Restricting $A$ to these subspaces gives sign det equal to -1 and 1 , respectively. This describes the virtual character $-\chi$.

Now we comment on the relationship between our equivariant orientation and the corresponding notion used by Lück and Rosenberg in [10]. Our work intersects with theirs when $Y$ is discrete, that is, we are dealing with a self-map with isolated, regular fixed points. Since both methods use induction in the same way, we will just consider the case when $Y$ is a point and $G$ is a finite group.

Thus $G$ acts by orthogonal transformations on a Euclidean space $E$, and $A$ is a $G$-equivariant, invertible linear map on $E$. We have shown above how to associate to this data a virtual character $\operatorname{sign}(A)$ of $G$, which is described in (8). To the same data, Lück and Rosenberg associate an invariant $\operatorname{Deg}_{0}^{G}(A)$ in the Abelian group $U^{G}(\star)$ of $\mathbb{Z}$-valued functions on the set $\Pi(G)$ of conjugacy classes of subgroups of $G$. Computations in [10] describe $\operatorname{Deg}_{0}^{G}(A)$ by the formula

$$
\operatorname{Deg}_{0}^{G}(A)(L)=\operatorname{sign} \operatorname{det}\left(\left.A\right|_{\text {Fix }(L)}\right) .
$$


Comparison with (8) shows that this restricts to our virtual character on cyclic subgroups. Thus their local invariant contains more information than ours.

\subsection{The smooth Lefschetz map}

Now let $X$ be a smooth Riemannian manifold and assume that $G$ acts on $X$ isometrically and continuously.

Let $\psi: X \rightarrow X$ be a $G$-equivariant self-map of $X$. In order to write down an explicit local formula for $\operatorname{Lef}(\psi)$, we impose the following restrictions on $\psi$ :

- $\psi$ is smooth;

- the fixed point subset $\operatorname{Fix}(\psi)$ of $\psi$ is a submanifold of $X$;

- if $(p, \xi) \in T X$ is fixed by the derivative $D \psi$, then $\xi$ is tangent to $\operatorname{Fix}(\psi)$.

The last two conditions are automatic if $\psi$ is isometric with respect to some Riemannian metric (not necessarily the given one) and hence if $\psi$ has finite order.

In the simplest case, $\psi$ and $\mathrm{id}_{X}$ are transverse, that is, $\mathrm{id}-D \psi$ is invertible at each fixed point of $\psi$; this implies that $\psi$ has isolated fixed points. While this situation can always be achieved in the non-equivariant case, we cannot expect transversality for non-discrete $G$ because the fixed point set must be $G$-invariant.

To describe the Lefschetz invariant, we abbreviate $Y:=\operatorname{Fix}(\psi)$. This is a closed submanifold of $X$ by assumption. Let $v$ be the normal bundle of $Y$ in $X$. Since the derivative $D \psi$ fixes the tangent space of $Y$, it induces a linear map $D_{v} \psi: v \rightarrow v$. By assumption, the map $\mathrm{id}_{v}-D_{v} \psi: v \rightarrow v$ is invertible.

Theorem 2 Let $X$ be a complete smooth Riemannian manifold, let $G$ be a locally compact group that acts on $X$ continuously and by isometries, and let $\psi: X \rightarrow X$ be a smooth self-map. Assume that the fixed point subset $Y:=\operatorname{Fix}(\psi)$ of $\psi$ is a submanifold of $X$ and that all tangent vectors of $X$ fixed by $D \psi$ are tangent to $Y$.

Let $v$ be the normal bundle of $Y$ in $X$ and let $D_{v} \psi: v \rightarrow v$ be induced by the derivative of $\psi$ as above. Let $r_{Y}: \mathscr{C}_{0}(X) \rightarrow \mathscr{C}_{0}(Y)$ be the restriction map and let $\operatorname{Eul}_{Y} \in \mathrm{KK}_{0}^{G}\left(\mathscr{C}_{0}(Y), \mathbb{1}\right)$ be the equivariant $\mathrm{K}$-homology class of the de Rham operator on $Y$. Then

$$
\operatorname{Lef}(\psi)=r_{Y} \otimes_{\mathscr{C}_{0}(Y)} \operatorname{sign}\left(\operatorname{id}_{v}-D_{\nu} \psi\right) \otimes_{\mathscr{C}_{0}(Y)} \operatorname{Eul}_{Y}
$$

Remark 1 It is shown in [5] that the equivariant Euler characteristic Eul $\operatorname{Eu}_{X}:=\operatorname{Lef}\left(\mathrm{id}_{X}\right)$ is the class of the de Rham operator on $X$. This justifies the notation Eul $_{Y}$ in Theorem 2. If $\psi$ is the identity map, then Theorem 2 reduces to this description of $\operatorname{Eul}_{X}$.

Now we make Theorem 2 more concrete in the special case where $\psi$ and $\mathrm{id}_{X}$ are transverse. Then the fixed point subset $Y$ is discrete. A discrete set is a manifold, and Theorem 2 describes its Euler characteristic: the de Rham operator on $Y$ is the zero operator on $L^{2}\left(\Lambda_{\mathbb{C}}^{*}\left(T^{*} X\right)\right)=\ell^{2}(Y)$, so that we have the class of the representation $\mathscr{C}_{0}(Y) \rightarrow \mathbb{K}\left(\ell^{2} Y\right)$ by pointwise multiplication operators.

The normal bundle $v$ to $Y$ in $X$ is the restriction of $T X$ to $Y$. For $p \in Y$, let $n_{p}$ be +1 if $\operatorname{id}_{T_{p} X}-D_{p} \psi$ preserves orientation, and -1 otherwise. The graded equivariant line 
bundle $\operatorname{sign}\left(\mathrm{id}_{v}-D_{\nu} \psi\right)$ in Theorem 2 is determined by pairs $\left(n_{p}, \chi_{p}\right)$ for $p \in Y$, where $n_{p}$ is the parity of the representation at $p$ and $\chi_{p}$ is a certain real-valued character $\chi_{p}: \operatorname{Stab}(p) \rightarrow\{-1,+1\}$ that depends on $\mathrm{id}_{T_{p} X}-D_{p} \psi$ and the representation of the stabiliser $\operatorname{Stab}(p) \subseteq G$ on $T_{p} X$ via the formula (8). Equivariance implies that $n_{p}$ is constant along $G$-orbits, whereas $\chi_{p}$ behaves like $\chi_{g \cdot p}=\chi_{p} \circ \operatorname{Ad}\left(g^{-1}\right)$. Let $\ell_{\chi}^{2}(G p)$ be the representation of $G \ltimes \mathscr{C}_{0}(G / \operatorname{Stab}(p))$ obtained by inducing the representation $\chi_{p}$ from $\operatorname{Stab}(p)$, and let $\mathscr{C}_{0}(X)$ act on $\ell_{\chi}^{2}(G p)$ by restriction to $G / \operatorname{Stab}(p)$. This defines a $G$-equivariant*-homomorphism

$$
\xi_{G p, \chi}: \mathscr{C}_{0}(X) \rightarrow \mathbb{K}\left(\ell_{\chi}^{2} G\right)
$$

Theorem 2 implies

Corollary 1 If the graph of $\phi$ is transverse to the diagonal in $X \times X$, then

$$
\operatorname{Lef}(\psi)=\sum_{G p \in G \backslash \operatorname{Fix}(\psi)} n_{p}\left[\xi_{G p, \chi}\right]
$$

where $\left[\xi_{G p, \chi}\right] \in \mathrm{KK}_{0}^{G}\left(\mathscr{C}_{0}(X), \mathbb{1}\right)$ and the multiplicities $n_{p}$ are explained above.

Furthermore, the character $\chi: \operatorname{Stab}_{G}(p) \rightarrow\{-1,+1\}$ at a fixed point $p$ has the explicit formula

$$
\chi(g)=\operatorname{sign} \operatorname{det}\left(\mathrm{id}-D_{p} \phi\right) \cdot \operatorname{sign} \operatorname{det}\left(\mathrm{id}-\left.D_{p} \phi\right|_{\mathrm{Fix}(g)}\right) .
$$

If, in addition, $G$ is trivial and $X$ is connected, then $\xi_{G p, \chi}=\operatorname{ev}_{p}$ for all $p \in Y$; moreover, all point evaluations have the same K-homology class because they are homotopic. Hence we get the classical Lefschetz data multiplied by the K-homology class of a point

$$
\operatorname{Lef}(\psi)=\left(\sum_{p \in \operatorname{Fix}(\psi)} \operatorname{sign}\left(\operatorname{id}_{T_{p} X}-D \psi_{p}\right)\right) \cdot[\mathrm{ev}]
$$

as asserted above. This sum is finite if $X$ is compact.

Lemma 3 Let $H \subseteq G$ be compact and open, let $p, q \in X^{H}$ belong to the same path component of the fixed point subspace $X^{H}$, and let $\chi \in \operatorname{Rep}(H)$. Then

$$
\left[\xi_{G p, \operatorname{ind}_{H}^{\mathrm{Stab}(G p)}(\chi)}\right]=\left[\xi_{G q, \operatorname{ind}_{H}^{\mathrm{Stab}(G q)}(\chi)}\right] \quad \text { in } \mathrm{KK}_{0}^{G}\left(\mathscr{C}_{0}(X), \mathbb{1}\right)
$$

Proof The two representations $\xi_{G p, \ldots}$ and $\xi_{G q, \ldots}$ both act on $G$-Hilbert spaces that are isomorphic to $\operatorname{ind}_{H}^{G} \chi$. A homotopy from $p$ to $q$ inside $X^{H}$ provides a path of $G$-equivariant*-homomorphisms

$$
\mathscr{C}_{0}(X) \rightarrow \mathscr{C}_{0}(G / H) \rightarrow \mathbb{K}\left(\operatorname{ind}_{H}^{G} \chi\right)
$$


Example 4 Let $X \subseteq \mathbb{C}$ be the unit circle and let $G=\mathbb{Z} / 2=\{1, g\}$ act on $X$ by the conjugation $z \mapsto \bar{z}$, that is, reflection at the real axis. Let $n \in \mathbb{N}_{\geq 2}$ and consider the $G$-equivariant self-map $\psi(z):=z^{n}$ of $X$. Its fixed point set is the set of roots of unity of order $n-1$. The derivative $D \psi$ is $n$ at all fixed points, so that $\operatorname{id}_{\mathbb{R}}-D \psi=1-n$ is a negative multiple of the identity map on $\mathbb{R}$.

Let $\tau$ and $\chi$ be the trivial and non-trivial representations of $G$ and let $\mathbb{C}_{\tau}$ and $\mathbb{C}_{\chi}$ be $\mathbb{C}$ with the corresponding representation of $G$. If $p \in X$ is a $\psi$-fixed point different from \pm 1 , then its stabiliser in $G$ is trivial, so that there is no representation $\chi_{p}$ to worry about. By Example 2, each of these fixed points contributes $-\left[\xi_{\{z, \bar{z}\}}\right]$, where $\xi_{\{z, \bar{z}\}}$ is the representation of $\mathscr{C}_{0}(X)$ on $\ell^{2}(\{z, \bar{z}\})$ by pointwise multiplication. Example 3 shows that the contribution of 1 is $-\left[\xi_{\{1\}, \chi}\right]$; this is just the class of the representation of $\mathscr{C}_{0}(X)$ on $\mathbb{C}_{\chi}$ by evaluation at $1 \in X$. If $n$ is odd, then there is a similar contribution $-\left[\xi_{\{-1\}, \chi}\right]$ from the fixed point -1 .

The limit of $\xi_{\{z, \bar{z}\}}$ for $z \rightarrow \pm 1$ is $\left[\xi_{\{1\}, \chi \oplus \tau}\right]$ because $\chi \oplus \tau$ is the regular representation of $G$, and by Lemma 3 . Hence the contributions of \pm 1 are $\left[\xi_{ \pm 1, \tau}\right]-\left[\xi_{\{z, \bar{z}\}}\right]$ for any $z \in X \backslash\{ \pm 1\}$. We may abbreviate $\xi_{ \pm 1, \tau}=\mathrm{ev}_{ \pm 1}$. Thus we get

$$
\operatorname{Lef}(\psi)= \begin{cases}{\left[\mathrm{ev}_{1}\right]+\left[\mathrm{ev}_{-1}\right]-\frac{n+1}{2}\left[\xi_{\{z, \bar{z}\}}\right]} & \text { if } n \text { is odd } \\ {\left[\mathrm{ev}_{1}\right]-\frac{n}{2}\left[\xi_{\{z, \bar{z}\}}\right]} & \text { if } n \text { is even }\end{cases}
$$

We may also triangulate $X$ by taking \pm 1 as vertices and the upper and lower semicircles as edges. The combinatorial Lefschetz invariant of $\psi$ is given by the same formula in this case, as it should be. When we forget the $G$-action, $\operatorname{Lef}(\psi)$ simplifies to $(1-n) \cdot[\mathrm{ev}]$; this is the expected result because $1-n$ is the supertrace of the action of $\psi$ on the homology of $X$.

Example 5 Let $G \cong \mathbb{Z} \rtimes \mathbb{Z} / 2 \mathbb{Z}$ be the infinite dihedral group, identified with the group of affine transformations of $\mathbb{R}$ generated by $u(x)=-x$ and $w(x)=x+1$. Then $G$ has exactly two conjugacy classes of finite subgroups, each isomorphic to $\mathbb{Z} / 2$. Its action on $\mathbb{R}$ is proper, and the closed interval $[0,1 / 2]$ is a fundamental domain. There are two orbits of fixed point in $\mathbb{R}$ - those of 0 and $1 / 2$ - and their stabilisers represent the two conjugacy classes of finite subgroups.

Now we use some notation from Example 3. Each copy of $\mathbb{Z} / 2$ acting on the tangent space at the fixed point acts by multiplication by -1 on tangent vectors. Therefore, the computations in Example 3 show that for any nonzero real number $A$, viewed as a linear transformation of the tangent space that commutes with $\mathbb{Z} / 2$, we have

$$
\operatorname{sign}(A)= \begin{cases}\mathbb{R}_{\chi}^{\text {op }} & \text { if } A<0, \text { and } \\ \mathbb{R}_{\tau} & \text { if } A>0 .\end{cases}
$$

Let $\phi$ be a small $G$-equivariant perturbation of the identity map $\mathbb{R} \rightarrow \mathbb{R}$ with the following properties. First, $\phi$ maps the interval $[0,1 / 2]$ to itself. Second, its fixed points in $[0,1 / 2]$ are $1 / 4$ and the end points 0 and $1 / 2$; third, its derivative is bigger than 1 at both endpoints and between 0 and 1 at 1/4. Such a map $\phi$ clearly exists. Furthermore, it is homotopic to the identity map, so that $\operatorname{Lef}(\phi)=\operatorname{Eul}_{\mathbb{R}}$. 
By construction, there are three fixed points modulo $G$, namely, the orbits of $0,1 / 4$ and $1 / 2$. The isotropy groups of the first and third orbit are non-conjugate subgroups isomorphic to $\mathbb{Z} / 2$; from Example 3, each of them contributes $\mathbb{R}_{\chi}^{\text {op }}$. The point $1 / 4$ contributes the trivial character of the trivial subgroup. Hence

$$
\operatorname{Lef}(\phi)=-\left[\xi_{\mathbb{Z}, \chi}\right]-\left[\xi_{\mathbb{Z}+1 / 2, \chi}\right]+\left[\xi_{\mathbb{Z}+1 / 4}\right]
$$

On the other hand, suppose we change the above map $\phi$ to fix the same points but to have zero derivative at 0 and $1 / 2$ and large derivative at $1 / 4$. This is obviously possible. Then we get contributions of $\mathbb{R}_{\tau}$ at 0 and $1 / 2$ and a contribution of $-\left[\xi_{1 / 4}\right]$ at $1 / 4$. Hence

$$
\operatorname{Lef}(\phi)=\left[\xi_{\mathbb{Z}, \tau}\right]+\left[\xi_{\mathbb{Z}+1 / 2, \tau}\right]-\left[\xi_{\mathbb{Z}+1 / 4}\right]
$$

Combining both formulas yields

$$
\left[\xi_{\mathbb{Z}, \tau}\right]+\left[\xi_{\mathbb{Z}+1 / 2, \tau}\right]-\left[\xi_{\mathbb{Z}+1 / 4}\right]=-\left[\xi_{\mathbb{Z}, \chi}\right]-\left[\xi_{\mathbb{Z}+1 / 2, \chi}\right]+\left[\xi_{\mathbb{Z}+1 / 4}\right]
$$

By the way, the left-hand side is the description of $\operatorname{Eul}_{\mathbb{R}}$ we get from the combinatorial dual with the obvious $G$-invariant triangulation of $\mathbb{R}$ with vertex set $\mathbb{Z} \cdot 1 / 2 \subset \mathbb{R}$.

It is possible, of course, to verify (9) directly. Indeed, since $\tau+\chi$ is the regular representation $\lambda,(9)$ is equivalent to

$$
\left[\xi_{\mathbb{Z}, \lambda}\right]+\left[\xi_{\mathbb{Z}+1 / 2, \lambda}\right]=2\left[\xi_{\mathbb{Z}+1 / 4}\right]
$$

and this relation follows from Lemma 3.

Now let $G$ be a Lie group acting properly and isometrically on a Riemannian manifold $X$. Assume that $G \backslash X$ is compact. Let $\psi: X \rightarrow X$ be a smooth map. Assume that $F:=\operatorname{Fix}(\psi)$ is a manifold and the restriction of id $-D_{p} \psi$ to the normal bundle of $T_{p} F \subseteq T_{p} X$ is injective for all $p \in F$. Restriction of the Riemannian metric on $X$ to $F$ gives a Riemannian metric on $F$. Since $F$ is closed and $G \backslash X$ is compact, $G \backslash F$ is compact. Hence $G \backslash F$ is complete with respect to the restricted metric.

Let $H$ be a Lie group acting isometrically by orientation-preserving maps on an odd-dimensional complete Riemannian manifold $F$. Then $\operatorname{Eul}_{F} \cong 0$ (see [5]). Using Theorem 2, we see that odd-dimensional components of $G \backslash F$ do not contribute to Lef $(\psi)$. Only even-dimensional components may have non-trivial Euler characteristics.

Example 6 Let $\psi: \mathbb{S}^{3} \rightarrow \mathbb{S}^{3}$ be the reflection $\psi(x, y, z, w):=(x, y, z,-w)$, where

$$
\mathbb{S}^{3}:=\left\{(x, y, z, w) \in \mathbb{R}^{4} \mid x^{2}+y^{2}+z^{2}+w^{2}=1\right\} .
$$

The group $\mathrm{O}(3, \mathbb{R}) \subset \mathrm{O}(4, \mathbb{R})$ acts on $\mathbb{S}^{3}$ commuting with $\psi$, so that Theorem 2 applies. We have $\operatorname{Fix}(\psi) \cong \mathbb{S}^{2}$ and

$$
\operatorname{Lef}(\psi)=-i_{*}\left(\operatorname{Eul}_{\mathbb{S}^{2}}\right) \in \mathrm{KK}_{0}^{\mathrm{O}(3, \mathbb{R})}\left(\mathscr{C}\left(\mathbb{S}^{3}\right), \mathbb{C}\right)
$$


where $i: \mathbb{S}^{2} \rightarrow \mathbb{S}^{3}$ is the inclusion as the equator. The bundle sign(id $\left.-D \psi\right)$ is the trivial line bundle with trivial action of $\mathrm{O}(3, \mathbb{R})$, with negative parity; this accounts for the sign. Note that $\mathrm{Eul}_{\mathbb{S}^{2}}$ with respect to the group $\mathrm{O}(3, \mathbb{R})$ is nonzero because the (non-equivariant) Euler characteristic of $\mathbb{S}^{2}$ is 2 . The equivariant index of Eul $\mathbb{S}^{2}$ in $\mathrm{KK}_{0}^{\mathrm{O}(3, \mathbb{R})}(\mathbb{C}, \mathbb{C})$ is given by the character $\tau+$ det, where $\tau$ is the trivial character and det $: \mathrm{O}(3, \mathbb{R}) \rightarrow\{-1,+1\}$ is the determinant. This follows from the usual description of the index of the de Rham operator in terms of spaces of harmonic forms.

Finally, at least in the non-equivariant case, we record that maps of non-compact $G$-spaces usually have zero Lefschetz invariants:

Corollary 2 In the situation of Theorem 2, assume that $G$ is the trivial group, $\psi$ and $\mathrm{id}_{X}$ are transverse, and none of the connected components of $X$ are compact. Then $\operatorname{Lef}(\psi)=0$.

Proof There is an increasing sequence of compact subsets $\left(K_{m}\right)$ in $X$ such that, for each $m \in \mathbb{N}$, no component of $X \backslash K_{m}$ is compact. For each $p \in Y$, let $m$ be minimal with $p \in K_{m}$, and let $I_{p}$ be a path from $p$ to $\infty$ in $X^{+}$that does not meet $K_{m-1}$. This ensures that $\bigoplus_{p \in Y} \mathrm{ev}_{I_{p}(t)}$ is a homotopy from the *-homomorphism describing Lef $(\psi)$ to the zero map.

The equivariant Lefschetz map can carry information also for non-compact spaces. An analogue of Corollary 2 holds in the equivariant case for discrete $G$ provided for each finite subgroup $H \subseteq G$, the fixed point submanifold $\{x \in X \mid H x=x\}$ has no compact components; it suffices to assume this only for those components that contain a fixed point of $\psi$.

\subsection{The Lefschetz formula: comparing the two computations}

Theorem 3 Let $G$ be a discrete group and let $X$ be a smooth manifold equipped with a proper action of $G$. Let $\psi: X \rightarrow X$ be a smooth map that satisfies the conditions of Theorem 2. Choose a $G$-equivariant cellular decomposition of $X$, and let $\psi^{\prime}: X \rightarrow X$ be $G$-homotopic to $\psi$ and cellular. Then

$$
\begin{aligned}
\sum_{\dot{\sigma} \in G \backslash S X} \mathrm{n}(\Psi, \dot{\sigma})\left[\xi_{\dot{\sigma}}\right] & =\operatorname{Lef}\left(\psi^{\prime}\right) \\
& =\operatorname{Lef}(\psi)=r_{Y} \otimes_{\mathscr{C}_{0}(Y)} \operatorname{sign}\left(\mathrm{id}_{\nu}-D_{\nu} \psi\right) \otimes_{\mathscr{C}_{0}(Y)} \operatorname{Eul}_{Y}
\end{aligned}
$$

Here $S X$ denotes the set of cells in the decomposition, and the multiplicities $\mathrm{n}(\Psi, \dot{\sigma})$ and the equivariant $\mathrm{K}$-homology classes $\left[\xi_{\dot{\sigma}}\right]$ are as in Theorem $1 ; Y=\operatorname{Fix}(\psi), r_{Y}$ is the class of the restriction map $\mathscr{C}_{0}(X) \rightarrow \mathscr{C}_{0}(Y)$, $v$ is the normal bundle of $Y$, and Eul $_{Y}$ is the equivariant K-homology class of the de Rham operator on $Y$ as in Theorem 2.

Proof Since $G$ is discrete and acts properly, $X$ carries a $G$-equivariant triangulation (see [7]) and a complete $G$-invariant Riemannian metric. 
The combinatorial Lefschetz invariant $\sum_{\dot{\sigma} \in G \backslash S X} \mathrm{n}(\Psi, \dot{\sigma})\left[\xi_{\dot{\sigma}}\right]$ is independent of the cellular decomposition. Hence we may compute it using the cellular structure underlying a triangulation (our duality approach does not work for general $G$-CW-complexes). Any $G$-equivariant self-map of a $G$-CW-complex is $G$-equivariantly homotopic to a cellular $G$-equivariant self-map, so that $\psi^{\prime}$ exists. We have $\operatorname{Lef}\left(\psi^{\prime}\right)=\operatorname{Lef}(\psi)$ because the Lefschetz invariant is homotopy invariant. Now combine the formulas in Theorems 1 and 2 .

This result is similar to the Lefschetz Fixed Point Formula of [10]. There are two differences: first, we allow $\psi$ to have non-isolated fixed points and describe the local contributions of fixed points differently even in the isolated case; second, we compute an element of $\operatorname{KK}_{0}^{G}\left(\mathscr{C}_{0}(X), \mathbb{1}\right)$ instead of the universal equivariant homology theory considered in [10].

\section{Lefschetz invariants for simplicial complexes}

We first recall briefly the combinatorial Kasparov dual in [5]. This construction is suggested by work of Kasparov and Skandalis in [9]. In order to compute Euler characteristics in [5] we reconstructed the results of Kasparov and Skandalis in more explicit terms. We refer the reader to our previous article for details and some notation. We are going to use the combinatorial Kasparov dual to compute the Lefschetz invariant of a cellular self-map. This is a matter of plugging all the ingredients into (5) and simplifying the outcome.

\subsection{Description of the combinatorial dual}

Let $X$ be a simplicial complex and let $G$ be a locally compact group acting simplicially on $X$. Thus $G$ permutes the simplices of $X$, and the stabiliser of each simplex is an open subgroup of $G$. Since this forces the connected component of the identity in $G$ to act trivially, the group $G$ will usually be totally disconnected, but we do not need this assumption. Let $S_{d} X$ be the set of $d$-simplices in $X$ and $S X=\bigsqcup S_{d} X$. In particular, $S_{0} X$ is the set of vertices of $X$.

We assume throughout that $X$ is finite-dimensional, say of dimension at most $n$. Let $\underline{\mathbf{n}}:=\{1, \ldots, n\}$. An ( $n$-dimensional, $G$-invariant) colouring on $X$ is a map $\gamma: S_{0} X \rightarrow$ $\underline{\mathbf{n}}$ that is $G$-invariant and satisfies $\gamma(\sigma) \neq \gamma(\tau)$ whenever $\sigma, \tau \in S_{0} X$ are joined by an edge. Equivalently, if $\sigma \in S X$ is any simplex, then $\gamma$ restricts to an injective map on the set of vertices of $\sigma$. Coloured simplicial complexes are also called typed.

The barycentric subdivision of an $n$-dimensional simplicial complex admits a canonical $n$-dimensional colouring, so that we may assume without loss of generality that $X$ itself has such a colouring $\gamma$. Since $\gamma$ is $G$-invariant, a group element that maps a simplex to itself must fix it pointwise.

We use the $n$-dimensional affine space

$$
E:=\left\{\left(t_{0}, \ldots, t_{n}\right) \in \mathbb{R}^{n+1} \mid t_{0}+\cdots+t_{n}=1\right\}
$$


and the standard $n$-simplex

$$
\Sigma:=\left\{\left(t_{0}, \ldots, t_{n}\right) \in E \mid t_{i} \geq 0 \text { for } i=0, \ldots, n\right\}
$$

For a non-empty subset $f \subseteq \underline{\mathbf{n}}$, we let $\Sigma_{f} \subseteq \Sigma$ be the corresponding face; its points are characterised by $t_{j}=0$ for $j \notin f$. This subset is denoted by $|f|$ in [5]. The map $f \mapsto \Sigma_{f}$ gives a bijection between the set $\mathcal{S}(\underline{\mathbf{n}})$ of non-empty subsets of $\underline{\mathbf{n}}$ and the set of faces of $\Sigma$.

Let $\tau$ be a simplex in $X$ with vertices $v_{0}, \ldots, v_{d}$, where $d=\operatorname{dim} \tau$. We also write $|\tau|$ for the corresponding subset of $X$. Points in $|\tau|$ can be described by barycentric coordinates as $t_{0} v_{0}+\cdots+t_{d} v_{d}$ with $t_{0}, \ldots, t_{d} \in \mathbb{R}_{\geq 0}$ and $t_{0}+\cdots+t_{d}=1$. Since the colouring $\gamma$ is injective on the vertices of $\tau$, the set

$$
\gamma(\tau):=\left\{\gamma\left(v_{0}\right), \ldots, \gamma\left(v_{d}\right)\right\}
$$

has $d+1$ elements and hence determines a $d$-dimensional face $\Sigma_{\gamma(\tau)}$ of $\Sigma$. Even more, $\gamma$ induces a linear bijection

$$
|\gamma|:|\tau| \rightarrow \Sigma_{\gamma(\tau)}, \quad t_{0} v_{0}+\cdots+t_{d} v_{d} \mapsto t_{0} e_{\gamma\left(v_{0}\right)}+\cdots+t_{d} e_{\gamma\left(v_{d}\right)}
$$

Here $e_{i} \in \Sigma \subseteq E$ is the $i$ th standard basis vector.

We define

$$
\begin{aligned}
R_{f} & :=\left\{t \in E \mid t_{j} \geq 0 \text { for } j \in f \text { and } t_{j} \leq 0 \text { for } j \notin f\right\} . \\
R_{\leq f} & :=\bigcup_{l \subseteq f} R_{l}=\left\{t \in E \mid t_{j} \leq 0 \text { for } j \in \underline{\mathbf{n}} \backslash f\right\} .
\end{aligned}
$$

We introduce homogeneous coordinates by letting

$$
\left[t_{0}: \ldots: t_{n}\right]:=\frac{\left(t_{0}, \ldots, t_{n}\right)}{t_{0}+\cdots+t_{n}}
$$

for $\left(t_{0}, \ldots, t_{n}\right) \in \mathbb{R}^{n+1}$ with $t_{0}+\cdots+t_{n} \neq 0$. We define a retraction $q: E \rightarrow \Sigma$ from $E$ to $\Sigma$ by

$$
q(t):=\left[\max \left(t_{0}, 0\right): \ldots: \max \left(t_{n}, 0\right)\right] \quad \text { for } t=\left(t_{0}, \ldots, t_{n}\right) \in E
$$

Thus $R_{\leq f}=q^{-1}\left(\Sigma_{f}\right)$ for $f \in \mathcal{S}(\underline{\mathbf{n}})$.

Let $\mathbb{K}:=\mathbb{K}\left(\ell^{2} S X\right)$. If $T \in \mathbb{K}$, let $T_{\sigma, \sigma^{\prime}}$ for $\sigma, \sigma^{\prime} \in S X$ be its matrix coefficients.

Definition 4 The dual $\mathrm{C}^{*}$-algebra of $X$ is defined by

$$
\mathscr{P}:=\left\{\varphi \in \mathscr{C}_{0}(E, \mathbb{K}) \mid \operatorname{supp} \varphi_{\sigma, \sigma^{\prime}} \subseteq R_{\leq \gamma\left(\sigma \cap \sigma^{\prime}\right)} \text { for all } \sigma, \sigma^{\prime} \in S X\right\}
$$


Example 7 Suppose $X$ is a single 1-simplex-together with its two vertices. Thus $S X$ has three elements. The dual $C^{*}$-algebra is the algebra of $3 \times 3$-matrices with a pattern of entries of the form

$$
\left(\begin{array}{ccc}
\mathscr{C}_{0}\left(\mathbb{R}_{<0}\right) & \mathscr{C}_{0}\left(\mathbb{R}_{<0}\right) & 0 \\
\mathscr{C}_{0}\left(\mathbb{R}_{<0}\right) & \mathscr{C}_{0}(\mathbb{R}) & \mathscr{C}_{0}\left(\mathbb{R}_{>1}\right) \\
0 & \mathscr{C}_{0}\left(\mathbb{R}_{>1}\right) & \mathscr{C}_{0}\left(\mathbb{R}_{>1}\right)
\end{array}\right)
$$

This $C^{*}$-algebra is Morita-Rieffel equivalent to $\mathscr{C}_{0}(\mathbb{R})$. This is to be expected because $[0,1]$ is homotopy equivalent to the point, so that its dual should be, up to dimension shift, KK-equivalent to $\mathbb{1}$.

We return to the general case. The pointwise multiplication homomorphism $\mathscr{C}_{0}(E) \otimes$ $\mathscr{P} \rightarrow \mathscr{P}$ turns $\mathscr{P}$ into a $C^{*}$-algebra over $E$. To describe the fibre at $t \in E$, let $f:=\left\{j \in \underline{\mathbf{n}} \mid t_{j} \geq 0\right\}$; equivalently, this is the minimal subset of $\underline{\mathbf{n}}$ for which $t$ is contained in the interior of $R_{\leq f}$. Let $S X_{\geq f}:=\{\sigma \in S X \mid \gamma(\sigma) \supseteq f\}$ and define a relation on $S X_{\geq f}$ by

$$
\sigma \sim_{f} \sigma^{\prime} \Longleftrightarrow \gamma\left(\sigma \cap \sigma^{\prime}\right) \supseteq f
$$

This is an equivalence relation because $\gamma$ is a colouring. The fibre of $\mathscr{P}$ over $t$ is the $\mathrm{C}^{*}$-algebra of the equivalence relation $\sim_{f}$ on $S X_{\geq f}$ or, equivalently,

$$
\mathbb{K}_{f}:=\left\{\varphi \in \mathbb{K} \mid \varphi_{\sigma, \sigma^{\prime}}=0 \text { unless } \gamma\left(\sigma \cap \sigma^{\prime}\right) \supseteq f\right\} .
$$

This is a direct sum of matrix algebras with one summand for each simplex in $X$ of colour $f$.

To be part of a Kasparov dual, $\mathscr{P}$ must be a $C^{*}$-algebra over $X$. This structure can also be described by a non-degenerate $G$-equivariant *-homomorphism $m: \mathscr{C}_{0}(X, \mathscr{P}) \cong \mathscr{C}_{0}(X) \otimes \mathscr{P} \rightarrow \mathscr{P}$. We recall its description in [5, Eq. 42]:

$$
m(\varphi)_{\sigma \sigma^{\prime}}(t)=\varphi_{\sigma \sigma^{\prime}}\left(\bar{q}_{\sigma}(t), t\right)
$$

for all $\varphi \in \mathscr{C}_{0}(X, \mathscr{P}), t \in E$, and, $\sigma, \sigma^{\prime} \in S X$. This involves the map

$$
\bar{q}: E \times S X \rightarrow X, \quad(t, \sigma) \mapsto \bar{q}_{\sigma}(t)
$$

which is defined as follows. If $\left.\varphi_{\sigma \sigma^{\prime}}(\lrcorner, t\right): X \rightarrow \mathbb{C}$ is not identically zero, then $t$ must lie in the interior of $R_{\leq \gamma\left(\sigma \cap \sigma^{\prime}\right)} \subseteq R_{\leq \gamma(\sigma)}$, so that we only need $\bar{q}_{\sigma}(t)$ for $t \in R_{\leq \gamma}(\sigma)$. Then $q(t) \in \Sigma_{\gamma(\sigma)}$, which is identified with $|\sigma| \subseteq X$ by the colouring. We let $\bar{q}_{\sigma}(t)$ be the point of $|\sigma|$ that corresponds to $q(t)$.

To compute the Lefschetz invariant of a self-map $\psi$, we must combine the multiplication map $m: \mathscr{C}_{0}(X, \mathscr{P}) \rightarrow \mathscr{P}$ with $\psi$ (see Lemma 1). The resulting *-homomorphism $\mu_{\psi}: \mathscr{C}_{0}(X) \otimes \mathscr{P} \rightarrow \mathscr{P}$ in (5) is given by

$$
\mu_{\psi}(\varphi)_{\sigma, \sigma^{\prime}}(t)=\varphi\left(\psi \circ \bar{q}_{\sigma}(t), t\right)_{\sigma, \sigma^{\prime}}
$$


Next we describe the class $D \in \mathrm{KK}_{n}^{G}(\mathscr{P}, \mathbb{1})$ for the Kasparov dual. Let

$$
\beta_{E} \in \mathrm{KK}_{n}^{G}\left(\mathscr{C}_{0}(E), \mathbb{1}\right) \text { and } \widehat{\beta}_{E}=\beta_{E}^{-1} \in \mathrm{KK}_{-n}^{G}\left(\mathbb{1}, \mathscr{C}_{0}(E)\right)
$$

be the Bott class and its inverse and let $i: \mathscr{P} \rightarrow \mathscr{C}_{0}(E) \otimes \mathbb{K}$ be the obvious inclusion. The latter defines a class $[i] \in \mathrm{KK}_{0}^{G}\left(\mathscr{P}, \mathscr{C}_{0}(E)\right)$ because $\mathrm{KK}^{G}$ is stable. We let

$$
D:=[i] \otimes_{\mathscr{C}_{0}(E)} \beta_{E} \in \mathrm{KK}_{n}^{G}(\mathscr{P}, \mathbb{1})
$$

The final datum $\Theta$ is more involved and uses two ingredients:

1. A $G$-equivariant continuous map $v$ from $X$ to the space of unit vectors in $\ell^{2}(S X)$, and

2. A family of *-homomorphisms $h_{s} !: \mathscr{C}_{0}(E) \rightarrow \mathscr{C}_{0}(E)$ for $s \in E$.

The map $v$ has two main features: first, the support of $v(x) \in \ell^{2}(S X)$ is the set of faces of $\sigma$ if $x \in|\sigma|$; second, $v(x)=\delta_{\sigma}$ if $x \in|\sigma|$ and $|\gamma|(x) \in C R_{\gamma(\sigma)}$, where the regions $C R_{f}$ are defined by

$$
C R_{f}:=\left\{t \in \Sigma \mid t_{i} \geq L \text { for } i \in f \text { and } t_{i} \leq L \text { for } i \in \underline{\mathbf{n}} \backslash f\right\}
$$

with an auxiliary parameter $L>0$. The subsets $C R_{f}$ for $f \in \mathcal{S}(\underline{\mathbf{n}})$ cover $\Sigma$. Each $C R_{f}$ is a closed polygonal neighbourhood of the barycentre of $\Sigma_{f}$ in $\Sigma$. The shape of these regions for $n=2$ is indicated in [5, Figure 3].

Passing from unit vectors to rank-1-projections, we get a map

$$
P: X \rightarrow \mathbb{K}, \quad x \mapsto|v(x)\rangle\langle v(x)|
$$

Let $x \in|\sigma|$. The properties of $v$ ensure two things. First, $P(x)_{\tau, \tau^{\prime}}=0$ unless both $\tau$ and $\tau^{\prime}$ are faces of $\sigma$. Second, if $|\gamma|(x) \in C R_{\gamma(\sigma)}$, then $P(x)_{\tau, \tau^{\prime}}=0$ unless $\tau=\tau^{\prime}=\sigma$, and $P(x)_{\sigma, \sigma}=1$.

The second ingredient for $\Theta$ is a family of *-homomorphisms

$$
h_{s} !: \mathscr{C}_{0}(E) \rightarrow \mathscr{C}_{0}(E)
$$

for $s \in E$, which is constructed as follows. Let

$$
B(\delta):=\left\{t \in \mathbb{R}^{n+1} \mid t_{0}+\cdots+t_{n}=0 \text { and }\left|t_{i}\right|<\delta \text { for } j=0, \ldots, n\right\} .
$$

Fix $\lambda>\frac{1}{1-(n+1) L}$ and let $r_{\lambda}: E \rightarrow E$ be the radial expansion by a factor of $\lambda$ around the barycentre of $\Sigma$ :

$$
r_{\lambda}\left(t_{0}, \ldots, t_{n}\right):=\left(\lambda t_{0}-\frac{\lambda-1}{n+1}, \ldots, \lambda t_{n}-\frac{\lambda-1}{n+1}\right) .
$$

By [5, Lemma 27], we can find $\delta>0$ such that

$$
r_{\lambda}(s)+B(\delta) \subseteq R_{\leq f} \quad \text { for all } f \in \mathcal{S}(\underline{\mathbf{n}}) \text { and } s \in C R_{f} .
$$


Let $h: E \stackrel{\rightrightarrows}{\rightarrow} B(\delta)$ be a fixed, orientation-preserving diffeomorphism. For $s \in E$, we define $h_{s} !: \mathscr{C}_{0}(E) \rightarrow \mathscr{C}_{0}(E)$ by

$$
h_{s} !(\varphi)(t):= \begin{cases}\varphi\left(h^{-1}(t-s)\right) & \text { for } t-s \in B(\delta), \\ 0 & \text { otherwise. }\end{cases}
$$

The definitions above ensure that

$$
\vartheta_{x}(\varphi):=h_{|\gamma|(x)} !(\varphi) \otimes P(x)
$$

is a ${ }^{*}$-homomorphism from $\mathscr{C}_{0}(E)$ to $\mathscr{P}$ for all $x \in X$. Letting $x$ vary, we get a $G \ltimes X$-equivariant*-homomorphism

$$
\vartheta: \mathscr{C}_{0}(X \times E) \cong \mathscr{C}_{0}(X) \otimes \mathscr{C}_{0}(E) \rightarrow \mathscr{C}_{0}(X) \otimes \mathscr{P} \cong \mathscr{C}_{0}(X, \mathscr{P}) .
$$

This yields $[\vartheta] \in \operatorname{RKK}_{0}^{G}\left(X ; \mathscr{C}_{0}(E), \mathscr{P}\right)$.

Finally, $\Theta \in \operatorname{RKK}_{-n}^{G}(X ; \mathbb{1}, \mathscr{P})$ is defined by

$$
\Theta:=\widehat{\beta}_{E} \otimes_{\mathscr{C}_{0}(E)}[\vartheta],
$$

where $\widehat{\beta}_{E} \in \mathrm{KK}_{-n}^{G}\left(\mathbb{1}, \mathscr{C}_{0}(E)\right)$ is the generator of Bott periodicity.

The data $(\mathscr{P}, \Theta, D)$ defined above is a $G$-equivariant Kasparov dual for $X$ of dimension $-n$ by [5, Theorem 29].

\subsection{Computing the Lefschetz invariant}

We now compute the Lefschetz invariant of a $G$-equivariant self-map $\psi: X \rightarrow X$ using the simplicial dual described above. The map $\psi$ is $G$-equivariantly homotopic to a $G$-equivariant cellular map, that is, a map that preserves the filtration of $X$ by skeleta. Even more, by an equivariant version of the Simplicial Approximation Theorem, any self-map is $G$-equivariantly homotopic to a $G$-equivariant simplicial map $\psi^{\prime}: \operatorname{Sd} X \rightarrow X$, where $\operatorname{Sd} X$ denotes a sufficiently fine $G$-invariant subdivision of $X$ (since we allow the subdivision to get finer near infinity, this even works if $G$ does not act cocompactly on $X$ ). Since the Lefschetz invariant of a self-map is homotopy invariant, we may assume that $\psi$ has this special form from now on.

The map $\psi$ is a cellular map with respect to the cellular decomposition underlying the original simplicial structure on $X$, so that it induces a cellular chain map $\Psi: C_{\bullet}(X) \rightarrow C_{\bullet}(X)$. The matrix coefficient $\Psi_{\sigma \tau}$ of $\Psi$ for two simplices $\sigma, \tau$ counts how many of the simplices in the subdivision of $\tau$ are mapped onto $\sigma$, with a sign depending on whether the map $\left.\psi\right|_{|\tau|}:|\tau| \rightarrow|\sigma|$ preserves or reverses orientation.

The starting point for our computation is (5). We must plug in the ingredients we have constructed above. This yields

$$
\begin{aligned}
\operatorname{Lef}(\psi) & =\bar{\Theta} \otimes_{\mathscr{C}_{0}(X, \mathscr{P})}\left[\mu_{\psi}\right] \otimes \mathscr{P} D \\
& =\widehat{\beta}_{E} \otimes_{\mathscr{C}_{0}(E)}[\vartheta] \otimes_{\mathscr{C}_{0}(X, \mathscr{P})}\left[\mu_{\psi}\right] \otimes \mathscr{P}[i] \otimes_{\mathscr{C}_{0}(E)} \beta_{E}
\end{aligned}
$$


in $\mathrm{KK}_{0}^{G}\left(\mathscr{C}_{0}(X), \mathbb{1}\right)$. Here $\beta_{E}$ and $\widehat{\beta}_{E}$ are the Bott class and its inverse, and the remaining ingredients are given by ${ }^{*}$-homomorphisms

$$
\mathscr{C}_{0}(X) \otimes \mathscr{C}_{0}(E) \stackrel{\vartheta}{\rightarrow} \mathscr{C}_{0}(X, \mathscr{P}) \stackrel{\mu_{\psi}}{\rightarrow} \mathscr{P} \stackrel{i}{\rightarrow} \mathscr{C}_{0}(E, \mathbb{K})
$$

The maps $\vartheta$ and $\mu_{\psi}$ are described in (13) and (10), the map $i$ is just the embedding. The Kasparov product for *-homomorphisms agrees with the usual composition. Hence

$$
\operatorname{Lef}(\psi)=\widehat{\beta}_{E} \otimes_{\mathscr{C}_{0}(E)}\left[\Xi_{\psi}\right] \otimes_{\mathscr{C}_{0}(E)} \beta_{E}
$$

with the $G$-equivariant *-homomorphism

$$
\Xi_{\psi}:=i \circ \mu_{\psi} \circ \vartheta: \mathscr{C}_{0}(X \times E) \rightarrow \mathscr{C}_{0}(E) \otimes \mathbb{K}\left(\ell^{2} S X\right) .
$$

Equations (13) and (10) yield

$$
\Xi_{\psi}(\varphi)_{\sigma, \sigma^{\prime}}(t)=\varphi\left(\psi \circ \bar{q}_{\sigma}(t), h^{-1}\left(t-r_{\lambda} \circ|\gamma| \circ \psi \circ \bar{q}_{\sigma}(t)\right)\right) \cdot P_{\sigma, \sigma^{\prime}}\left(\psi \circ \bar{q}_{\sigma}(t)\right)
$$

for all $\sigma, \sigma^{\prime} \in S X, t \in E, \varphi \in \mathscr{C}_{0}(X \times E)$; this is understood to be 0 unless $t-r_{\lambda} \circ|\gamma| \circ \psi \circ \bar{q}_{\sigma}(t) \in B(\delta)$.

Lemma 4 Let $\varphi \in \mathscr{C}_{0}(X \times E), t \in E$, and $\sigma, \sigma^{\prime} \in S X$, and let $x:=\psi \circ \bar{q}_{\sigma}(t)$. Assume that $\Xi_{\psi}(\varphi)_{\sigma, \sigma^{\prime}}(t) \neq 0$. Then:

1. $t \in B(\delta)+r_{\lambda} \circ|\gamma|(x)$,

2. $\sigma=\sigma^{\prime}$,

3. $|\gamma|(x) \in \Sigma_{\gamma(\sigma)} \cap C R_{\gamma(\sigma)}$,

4. $t$ belongs to the interior of $R_{\gamma(\sigma)}$.

Proof The first claim follows immediately from the definition of $h_{s}$ ! in (12). The next two properties are more interesting.

Choose $f \subseteq \underline{\mathbf{n}}$ with $|\gamma|(x) \in C R_{f}$. [5, Lemma 27] yields $t \in B(\delta)+r_{\lambda}(|\gamma|(x)) \subseteq$ $R_{\leq f}$. Thus $q(t) \in \Sigma_{f}$ and $\bar{q}_{\sigma}(t)$ belongs to the $d$-skeleton of $X$ with $d:=\operatorname{dim} f=$ $|\bar{f}|-1$.

Since $\psi$ is cellular, $x=\psi\left(\bar{q}_{\sigma}(t)\right)$ also belongs to the $d$-skeleton. Hence the point $s:=|\gamma|(x) \in \Sigma$ belongs to some $d$-dimensional face of $\Sigma$. Thus at most $d+1$ of its coordinates $s_{0}, \ldots, s_{n}$ can be non-zero. But $s \in C R_{f}$ means that $s_{j} \geq L$ for $j \in f$, providing $d+1$ non-zero coordinates. Hence $s_{j} \geq L$ for $j \in f$ and $s_{j}=0$ for $j \in \underline{\mathbf{n}} \backslash f$ or, equivalently, $s \in \Sigma_{f} \cap C R_{f}$. But then $P(x)$ is the projection onto the basis vector $\delta_{\tau}$, where $\tau$ is the unique simplex in $X$ with $x \in \tau$ and $\gamma(\tau)=f$. Thus $\tau=\sigma=\sigma^{\prime}$ and $f=\gamma(\sigma)$. We also get $|\gamma|(x)=s \in \Sigma_{\gamma(\sigma)} \cap C R_{\gamma(\sigma)}$ as asserted in (3). We have already seen above that $t=\left(t_{0}, \ldots, t_{n}\right)$ must belong to $R_{\leq \gamma(\sigma)}$, that is, $t_{i} \leq 0$ for $i \in \underline{\mathbf{n}} \backslash f$. If $t_{i} \leq 0$ for some $i \in f$, then $\bar{q}_{\sigma}(t)$ belongs to the $d-1$-skeleton of $X$, which is impossible. Hence $t_{i}>0$ for all $i \in f$. Furthermore, $t \in B(\delta)+r_{\lambda}\left(\Sigma_{f}\right)$ implies $t_{i}<0$ for $i \in \underline{\mathbf{n}} \backslash f$. Thus $t$ is an interior point of $R_{f}$. 
As a result, the range of $\Xi_{\psi}$ is contained in the $C^{*}$-subalgebra

$$
\mathscr{C}_{0}(E \times S X) \cong \mathscr{C}_{0}(E) \otimes \mathscr{C}_{0}(S X) \subseteq \mathscr{C}_{0}(E) \otimes \mathbb{K}\left(\ell^{2} S X\right)
$$

of all operators that are diagonal on $\ell^{2}(S X)$ in the standard basis. Let

$$
\Xi_{\psi, \sigma}: \mathscr{C}_{0}(X \times E) \rightarrow \mathscr{C}_{0}(E)
$$

be the value at $\sigma \in S X$, so that $\boldsymbol{\Xi}_{\psi}=\left(\boldsymbol{\Xi}_{\psi, \sigma}\right)_{\sigma \in S X}$.

Given $t \in E$ and $\sigma \in S X$, we define $y:=\bar{q}_{\sigma}(t)$ and $x:=\psi(y)=\psi \circ \bar{q}_{\sigma}(t)$ and let $f:=\gamma(\sigma)$. Lemma 4 yields $\Xi_{\psi, \sigma}(\varphi)(t)=0$ unless $x \in|\sigma|$ and $|\gamma|(x) \in C R_{f}$. If $x \in|\sigma|$ and $|\gamma|(x) \in C R_{f}$, then

$$
\Xi_{\psi, \sigma}(\varphi)(t)=\varphi\left(x, h^{-1}\left(t-r_{\lambda} \circ|\gamma|(x)\right)\right)
$$

because $v(x)=\delta_{\sigma}$. We have $y \in|\sigma|$ because the range of $\bar{q}_{\sigma}$ is contained in $\sigma$. Thus $\Xi_{\psi, \sigma}(\varphi)$ vanishes identically unless there is $y \in|\sigma|$ with $\psi(y) \in|\sigma|$.

If $x \in|\sigma|$, then there is a canonical linear homotopy from $x$ to the barycentre $\xi_{\sigma}$. Deforming $\Xi_{\psi, \sigma}$ along this homotopy, we get the map $\Xi_{\psi, \sigma}^{\prime}: \mathscr{C}_{0}(X \times E) \rightarrow \mathscr{C}_{0}(E)$ defined by

$$
\Xi_{\psi, \sigma}^{\prime}(\varphi)(t)=\varphi\left(\xi_{\sigma}, h^{-1}\left(t-r_{\lambda} \circ|\gamma|(x)\right)\right)
$$

for $x \in|\sigma|$ and $|\gamma|(x) \in C R_{f}$, and 0 otherwise. Hence the families of maps $\left(\Xi_{\psi, \sigma}\right)_{\sigma \in S X}$ and $\left(\Xi_{\psi, \sigma}^{\prime}\right)_{\sigma \in S X}$ define the same class in $\mathrm{KK}_{0}^{G}\left(\mathscr{C}_{0}(X \times E), \mathscr{C}_{0}(E)\right)$.

The map $\Xi_{\psi, \sigma}^{\prime}$ is an exterior product of the evaluation map

$$
\mathscr{C}_{0}(X) \rightarrow \mathbb{1}, \quad \varphi \mapsto \varphi\left(\xi_{\sigma}\right),
$$

and a certain endomorphism $\Xi_{\psi, \sigma}^{\prime \prime}$ of $\mathscr{C}_{0}(E)$. Since $\psi$ is $G$-equivariant, $\Xi_{\psi, \sigma}^{\prime \prime}$ only depends on the orbit $\dot{\sigma}:=G \sigma$ of $\sigma$. Hence we get

$$
\operatorname{Lef}(\psi)=\sum_{\dot{\sigma} \in G \backslash S X}\left[\xi_{\dot{\sigma}}\right] \otimes\left(\beta_{E} \otimes_{\mathscr{C}_{0}(E)}\left[\boldsymbol{\Xi}_{\psi, \dot{\sigma}}^{\prime \prime}\right] \otimes \mathscr{C}_{0}(E) \widehat{\beta}_{E}\right)
$$

with $\left[\xi_{\dot{\sigma}}\right]$ as defined in (7). Since $\mathrm{KK}_{0}\left(\mathscr{C}_{0}(E), \mathscr{C}_{0}(E)\right) \cong \mathbb{Z}$ by Bott periodicity, the class $\left[\Xi_{\psi, \dot{\sigma}}^{\prime \prime}\right]$ cannot contribute more than a multiplicity. This is determined by the following lemma:

Lemma $5\left[\Xi_{\psi, \dot{\sigma}}^{\prime \prime}\right]=\mathrm{n}(\Psi, \dot{\sigma}) \cdot[\mathrm{id}]$ in $\mathrm{KK}_{0}\left(\mathscr{C}_{0}(E), \mathscr{C}_{0}(E)\right)$ with the number $\mathrm{n}(\Psi, \dot{\sigma})$ defined in Notation 1.

The proof of Lemma 5 will finish the proof of Theorem 1 and will occupy the remainder of this section. 
Fix $\sigma \in S X$ and let $d:=\operatorname{dim} \sigma$ and $f:=\gamma(\sigma) \in \mathcal{S}(\underline{\mathbf{n}})$. Let

$$
D_{\sigma}:=\left\{x \in|\sigma||| \gamma \mid(x) \in C R_{f}\right\}
$$

This is a closed neighbourhood of the barycentre of $\sigma$ that does not intersect the boundary of $\sigma$. Let

$$
D_{\sigma}^{\prime}:=\left\{y \in|\sigma| \mid \psi(y) \in D_{\sigma}\right\}
$$

Recall that $\psi$ is a simplicial map $\operatorname{Sd} X \rightarrow X$ for some subdivision of $X$. Let $\operatorname{Sd} \sigma$ be the set of $d$-dimensional simplices in the subdivision of $\sigma$. If $\tau \in \operatorname{Sd} \sigma$, then either $\psi$ maps $\tau$ bijectively onto $\sigma$ or $|\psi(\tau)| \cap|\sigma| \subseteq \partial|\sigma|$. Hence $|\tau|$ and $D_{\sigma}^{\prime}$ intersect if and only if $\psi(\tau)=\sigma$. As a result, $D_{\sigma}^{\prime}$ is a disjoint union of homeomorphic copies of $C R_{f}$, one for each simplex $\tau \in \operatorname{Sd} \sigma$ with $\psi(\tau)=\sigma$. Let $\tau_{1}, \ldots, \tau_{k}$ be a list of these simplices. For $j=0, \ldots, k$, define $\alpha_{j}: \mathscr{C}_{0}(E) \rightarrow \mathscr{C}_{0}(E)$ by

$$
\alpha_{j}(\varphi)(t):= \begin{cases}\varphi\left(h^{-1}\left(t-r_{\lambda} \circ|\gamma| \circ \psi \circ \bar{q}_{\sigma}(t)\right)\right) & \text { if } \bar{q}_{\sigma}(t) \in \tau_{j} \cap D_{\sigma}^{\prime} \\ 0 & \text { otherwise }\end{cases}
$$

Since the supports of the maps $\alpha_{j}$ are disjoint, we get

$$
\left[\Xi_{\psi, \dot{\sigma}}^{\prime \prime}\right]=\left[\alpha_{0}\right]+\cdots+\left[\alpha_{k}\right]
$$

Thus it remains to check that $\left[\alpha_{j}\right]=(-1)^{d} \varepsilon_{j}$, where $\varepsilon_{j}= \pm 1$ depending on whether $\left.\psi\right|_{\left|\tau_{j}\right|}:\left|\tau_{j}\right| \rightarrow|\sigma|$ preserves or reverses orientation.

We claim that the fixed point equation

$$
t=r_{\lambda} \circ|\gamma| \circ \psi \circ \bar{q}_{\sigma}(t)
$$

has a solution $t=\left(t_{0}, \ldots, t_{n}\right)$ in the interior of $R_{f}$ with $\bar{q}_{\sigma}(t) \in\left|\tau_{j}\right| \cap D_{\sigma}^{\prime}$. In particular, $\alpha_{j}$ is not identically zero.

We may assume right away that $t=r_{\lambda}(s)$ for some $s \in C R_{f} \cap \Sigma_{f}$. Thus

$$
\begin{array}{ll}
t_{i}>0 & \text { for } i \in f, \text { and } \\
t_{i}=-\frac{\lambda-1}{n+1} & \text { for } i \in \underline{\mathbf{n}} \backslash f .
\end{array}
$$

Although $\bar{q}_{\sigma}$ is non-linear in general because of the homogeneous coordinates involved, its restriction to points of this special form is linear: it simply annihilates the coefficients $t_{i}$ for $i \notin f$ and rescales the others by the constant

$$
\frac{(n+1) \lambda}{(d+1) \lambda+(n-d)}
$$

to get a point in $E$. Furthermore, $\bar{q}_{\sigma}$ is invertible between the relevant $d$-dimensional subspaces. We consider this restriction in the following when we speak of $\bar{q}_{\sigma}^{-1}$. Thus 
our fixed point problem is equivalent to finding $u:=\bar{q}_{\sigma}(t) \in\left|\tau_{j}\right| \cap D_{\sigma}^{\prime}$ with

$$
u=\bar{q}_{\sigma}^{-1} \circ r_{\lambda} \circ|\gamma| \circ \psi(u)
$$

The map $A:=\bar{q}_{\sigma}^{-1} \circ r_{\lambda} \circ|\gamma| \circ \psi$ is affine on $\left|\tau_{j}\right| \cap D_{\sigma}^{\prime}$ because each of the factors is affine on the relevant subsets. Furthermore, $A$ is expansive in all directions: the preimage of $|\sigma|$ is contained in the interior of $\left|\tau_{j}\right| \subseteq|\sigma|$. Hence its inverse map $A^{-1}$ is uniformly contractive. Thus $A^{-n}(s)$ converges towards a fixed point of $A$. This shows that a fixed point exists.

Bott periodicity implies that the inclusion map $\mathscr{C}_{0}(B(\delta)) \rightarrow \mathscr{C}_{0}\left(\mathbb{R}^{n}\right)$ is a KK-equivalence for all $\delta>0$. Hence we can compute the class of $\alpha_{j}$ by restricting it to an arbitrarily small ball around some point. Near the fixed point $t$ constructed above, the map

$$
t^{\prime} \mapsto h^{-1}\left(t^{\prime}-r_{\lambda} \circ|\gamma| \circ \psi \circ \bar{q}_{\sigma}\left(t^{\prime}\right)\right)
$$

is smooth. Hence $\left[\alpha_{j}\right]= \pm 1$ in $\mathrm{KK}_{0}\left(\mathscr{C}_{0}(E), \mathscr{C}_{0}(E)\right)$, where the sign is the sign of the determinant of the derivative of the above map. Since $h$ preserves orientation, we may omit it without changing the sign. In the directions orthogonal to $r_{\lambda}\left(\Sigma_{f}\right)$, the above map acts identically, so that they contribute no sign either. Thus the sign is the same as the sign of the linear map id $-A_{0}$, where $A_{0}$ is the linear part of the affine map $A$ above. Since $\left\|A_{0}^{-1}\right\|<1$ and id $-A_{0}=-A_{0} \cdot\left(\mathrm{id}-A_{0}^{-1}\right)$, this is the same as the sign of $\operatorname{det}\left(-A_{0}\right)=(-1)^{d} \operatorname{det}\left(A_{0}\right)$. Thus we get $(-1)^{d}$ if $\psi$ preserves orientation and $(-1)^{d+1}$ otherwise. This finishes the proof of Lemma 5 and thus of Theorem 1.

\section{Lefschetz invariants for smooth manifolds}

In this section, $X$ is a complete Riemannian manifold. The action of $G$ on $X$ is not quite required to be proper, but only isometric, or equivalently, the action should factor through a proper action. (The equivalence of these two conditions follows because the isometry group of $X$ is a Lie group that acts properly on $X$ provided $X$ has only finitely many connected components; conversely, given a proper action of a Lie group by diffeomorphisms, there is a complete Riemannian metric for which the action is isometric.) Such actions are those for which Kasparov originally proved duality results in [8, Sect. 4]. His construction uses Clifford algebras and differential operators. First we describe this dual. Then we compute the Lefschetz invariant of a suitable self-map. This is done once again by plugging all the ingredients into (5) and simplifying the result. To prepare for the main line of argument, we need a formula for the result of twisting the Thom isomorphism by a vector bundle automorphism. This is the source of the line bundle sign $\left(\mathrm{id}_{v}-D_{\nu} \psi\right)$ in our computation.

\subsection{Description of the Clifford algebra dual}

The complex Clifford algebras of the tangent spaces of $X$ form a locally trivial bundle of finite-dimensional $\mathbb{Z} / 2$-graded $C^{*}$-algebras $\operatorname{Cliff}\left(T^{*} X\right)$. Since $G$ acts isometrically 
on $X$, we get an induced action on $\operatorname{Cliff}\left(T^{*} X\right)$ by grading preserving *-algebra automorphisms. We let

$$
\mathscr{P}:=\Gamma_{0}\left(X, \operatorname{Cliff}\left(T^{*} X\right)\right)
$$

be the $\mathbb{Z} / 2$-graded $G$ - $C^{*}$-algebra of sections of $\operatorname{Cliff}\left(T^{*} X\right)$.

Let $\Lambda:=\Lambda_{\mathbb{C}}^{*}\left(T^{*} X\right)$ be the complexified exterior algebra bundle of $T^{*} X$. There is a canonical isomorphism $\operatorname{Cliff}\left(T^{*} X\right) \cong \Lambda$ that preserves the grading, inner products, and the $G$-action, but not the algebra structure. We let $c: \operatorname{Cliff}\left(T^{*} X\right) \rightarrow \operatorname{End}(\Lambda)$ be the resulting representation by Clifford multiplication.

We describe this on the level of forms. Let $\lambda_{\omega}: \Lambda \rightarrow \Lambda$ be the exterior product with $\omega \in \Lambda$. Let $i_{\omega}$ denote the interior product with $\omega$, that is,

$$
i_{\omega}\left(u_{1} \wedge \cdots \wedge u_{k}\right):=\sum_{j=1}^{k}(-1)^{j-1}\left\langle\omega, u_{j}\right\rangle u_{1} \wedge \cdots \wedge \widehat{u_{j}} \wedge \cdots \wedge u_{k}
$$

A simple calculation yields the graded commutator of these operations:

$$
i_{\omega} \lambda_{\tau}+\lambda_{\tau} i_{\omega}=\langle\omega, \tau\rangle
$$

In particular, $c(\omega):=\lambda_{\omega}+i_{\omega}$ satisfies $c(\omega)^{2}=\|\omega\|^{2}$. Since $i_{\omega}=\lambda_{\omega}^{*}$ as well, $c$ defines a representation of the Clifford algebra $\operatorname{Cliff}\left(T^{*} X\right)$ on $\Lambda$.

Let $\mathrm{d}$ be the usual boundary map on differential forms and let $\mathrm{D}_{\mathrm{dR}}:=\mathrm{d}+\mathrm{d}^{*}$. This is a $G$-equivariant self-adjoint, odd, elliptic differential operator of order 1 , and it commutes with $c(\mathscr{P})$ up to bounded operators. Now let $\mathscr{H}:=L^{2}(\Lambda)$; this is a $\mathbb{Z} / 2$-graded Hilbert space with a unitary representation of $G$, and $c$ yields a grading-preserving, $G$-equivariant ${ }^{*}$-representation of $\mathscr{P}$ on $\mathscr{H}$. The operator $\mathrm{D}_{\mathrm{dR}}$ is essentially selfadjoint, and $M_{f}\left(1+\mathrm{D}_{\mathrm{dR}}^{2}\right)^{-1}$ is compact for all $f \in \mathscr{C}_{0}(X)$ because $X$ is complete. Therefore, $\left(\mathscr{H}, c, \mathrm{D}_{\mathrm{dR}} /\left(1+\mathrm{D}_{\mathrm{dR}}^{2}\right)^{1 / 2}\right)$ is a Kasparov cycle for $\operatorname{KK}_{0}^{G}(\mathscr{P}, \mathbb{1})$, which we denote by $D$.

The diagonal embedding $X \rightarrow X \times X$ has a $G$-invariant tubular neighbourhood $U$ that is $G$-equivariantly diffeomorphic to the normal bundle $T X$ for the embedding. We can choose the diffeomorphism $\Phi: T X \rightarrow U$ of the form

$$
(x, \xi) \mapsto\left(x, \exp _{x}\left(\alpha_{x}(\|\xi\|) \cdot \xi\right)\right)
$$

for a function $\alpha: X \times \mathbb{R}_{\geq 0} \rightarrow \mathbb{R}$ that takes care of a possibly finite injectivity radius. It is important that $\pi_{1} \circ \bar{\Phi}$ is the usual projection $T X \rightarrow X$.

Let $J_{U} \subseteq \mathscr{C}_{0}(X) \otimes \mathscr{P}$ be the $G$-invariant ideal of functions that vanish outside $U$. We view this as a $G$-equivariant Hilbert module over $\mathscr{C}_{0}(X) \otimes \mathscr{P}$ in the usual way. We let $\mathscr{C}_{0}(X)$ act on $J_{U}$ by pointwise multiplication: $\left(f_{1} \cdot f_{2}\right)(x, y):=f_{1}(x) \cdot f_{2}(x, y)$. Identifying $U \cong T X$, we get a canonical section of the bundle underlying $J_{U}$, which associates to $\Phi(x, \xi) \in U$ the vector $\xi /\left(1+|\xi|^{2}\right)^{1 / 2} \in T_{X} X$ viewed as an element of Cliff $\left(T_{X} X\right)$. This defines a $G$-invariant, odd, self-adjoint multiplier $F$ of $J_{U}$ with $1-F^{2} \in J_{U}$. Hence $\left(J_{U}, F\right)$ yields a class $\Theta \in \operatorname{RKK}_{0}^{G}(X ; \mathbb{1}, \mathscr{P})$. 
Results in $[8$, Sect. 4] show that $(\mathscr{P}, \Theta, D)$ as defined above is a Kasparov dual for $X$.

\subsection{A twisted Thom isomorphism}

The computations in this section explain how the line bundle sign(id $\left.-D_{\nu} \psi\right)$ appears in our Lefschetz formula.

Let $G$ be a locally compact group, let $X$ be a locally compact proper $G$-space, and let $\pi: E \rightarrow X$ be a $G$-equivariant real vector bundle over $X$ with $G$-invariant inner product. First, we generalise the construction of $D$ and $\Theta$ above by working fibrewise. For each $x \in X$, the fibre $E_{x}$ has $\mathscr{C}_{0}\left(E_{x}, \operatorname{Cliff}\left(E_{x}\right)\right)$ as a Kasparov dual, via classes $D_{x}$ and $\Theta_{x}$. These combine to classes

$$
\begin{aligned}
& D_{E}^{X} \in \mathscr{R} \mathrm{KK}_{0}^{G}\left(X ; \mathscr{C}_{0}(E, \operatorname{Cliff}(E)), \mathscr{C}_{0}(X)\right), \\
& \Theta_{E}^{X} \in \mathscr{R} \mathrm{KK}_{0}^{G}\left(E ; \mathscr{C}_{0}(X), \mathscr{C}_{0}(E, \operatorname{Cliff}(E))\right) ;
\end{aligned}
$$

here we tacitly pull back $E$ to a bundle on $E$ via $\pi: E \rightarrow X$ to form

$$
\mathscr{P}_{E}^{X}:=\mathscr{C}_{0}(E, \operatorname{Cliff}(E))
$$

this is a $G-C^{*}$-algebra over $E$.

More explicitly, $D_{E}^{X}$ is the de Rham operator along the fibres of $\pi$. Since $E$ is already a vector bundle over $X$, all of $E \times_{X} E$ is a tubular neighbourhood of the diagonal $E \subseteq E \times{ }_{X} E$. Hence we can simplify $\Theta_{E}^{X}$; the underlying Hilbert module is simply

$$
\mathscr{C}_{0}\left(E \times_{X} E, \pi_{2}^{*} \operatorname{Cliff}(E)\right)
$$

where $\pi_{2}: E \times_{X} E \rightarrow E$ is the second coordinate projection. The group $G$ acts in an obvious way, and $\mathscr{C}_{0}(E)$ acts by pointwise multiplication via $\pi_{1}: E \times_{X} E \rightarrow E$. We define an essentially unitary multiplier of $\mathscr{C}_{0}\left(E \times_{X} E, \pi_{2}^{*} \operatorname{Cliff}(E)\right)$, that is, a bounded continuous section of $\pi_{2}^{*} \operatorname{Cliff}(E)$, by

$$
F(\xi, \eta):=\left(1+\|\xi-\eta\|^{2}\right)^{-1 / 2} \cdot(\xi-\eta) .
$$

This determines an operator $F$ on $\mathscr{C}_{0}\left(E \times_{X} E, \pi_{2}^{*} \mathrm{Cliff}(E)\right)$, and we get our cycle $\Theta_{E}^{X}$. The triple $\left(\mathscr{P}_{E}^{X}, D_{E}^{X}, \Theta_{E}^{X}\right)$ is a $G$-equivariant Kasparov dual for the space $E$ over $X$ (see [6] for this relative notion of duality). This means that we have canonical isomorphisms

$$
\mathscr{R} \mathrm{KK}_{*}^{G}\left(E ; \pi^{*}(A), \pi^{*}(B)\right) \cong \mathscr{R} \mathrm{KK}_{*}^{G}\left(X ; \mathscr{C}_{0}(E, \operatorname{Cliff}(E)) \otimes_{X} A, B\right)
$$


for all $G \ltimes X-C^{*}$-algebras $A$ and $B$. Actually, since the projection $E \rightarrow X$ is a $G$-equivariant homotopy equivalence,

$$
\mathscr{R} \mathrm{KK}_{*}^{G}\left(E ; \pi^{*}(A), \pi^{*}(B)\right) \cong \mathscr{R} \mathrm{KK}_{*}^{G}(X ; A, B)
$$

The Kasparov duality simply means that $D_{E}^{X} \in \mathscr{R} \mathrm{KK}_{*}^{G}\left(X ; \mathscr{P}_{E}^{X}, \mathscr{C}_{0}(X)\right)$ is invertible and that the inverse is the element

$$
\tilde{\Theta}_{E}^{X} \in \mathscr{R} \mathrm{KK}_{*}^{G}\left(X ; \mathscr{C}_{0}(X), \mathscr{P}_{E}^{X}\right)
$$

that corresponds to $\Theta_{E}^{X}$. Explicitly, the underlying Hilbert module of $\tilde{\Theta}_{E}^{X}$ is $\mathscr{P}{ }_{E}^{X}$ with the usual action of $G$ and the representation of $\mathscr{C}_{0}(X)$ by pointwise multiplication operators. The essentially unitary operator in the Kasparov cycle is the multiplier $F_{E}^{X}$ of $\mathscr{P}_{E}^{X}$ defined by

$$
E \ni(x, \xi) \mapsto\left(1+\|\xi\|^{2}\right)^{-1 / 2} \xi \in E_{x} \subseteq \operatorname{Cliff}\left(E_{x}\right)
$$

The proof that $D_{E}^{X}$ and $\tilde{\Theta}_{E}^{X}$ are inverse to each other can be reduced to the case where $X$ is a point using the same trick as in [11, Théorème 7.4]. (The Clifford algebras allow a kind of Thom isomorphism even if $E$ is not $\mathrm{K}$-oriented).

The following proposition is the entry point for the line bundle $\operatorname{sign}(f)$ in our Lefschetz computation. It is a refinement of the results in [4, Sect. 2].

Proposition 1 Let $f: E \rightarrow E$ be a G-equivariant isomorphism of vector bundles. Let $\left[f^{!}\right] \in \mathscr{R} \mathrm{KK}_{0}^{G}\left(X ; \mathscr{P}_{E}^{X}, \mathscr{P}_{E}^{X}\right)$ be the class of

$$
f^{!}: \mathscr{C}_{0}(E, \operatorname{Cliff}(E)) \rightarrow \mathscr{C}_{0}(E, \operatorname{Cliff}(E)), \quad f^{!}(\varphi)(x, \xi):=\varphi(x, f(\xi))
$$

for $(x, \xi) \in E, \varphi \in \mathscr{C}_{0}(E, \operatorname{Cliff}(E))=\mathscr{P}_{E}^{X}$. The composition

$$
\mathscr{C}_{0}(X) \stackrel{\tilde{\Theta}_{E}^{X}}{\longrightarrow} \mathscr{P}_{E}^{X} \stackrel{\left[f^{!}\right]}{\longrightarrow} \mathscr{P}_{E}^{X} \stackrel{D_{E}^{X}}{\longrightarrow} \mathscr{C}_{0}(X)
$$

is the class in $\mathrm{KK}_{0}^{G}\left(\mathscr{C}_{0}(X), \mathscr{C}_{0}(X)\right)$ of the $G$-equivariant $\mathbb{Z} / 2$-graded line bundle $\operatorname{sign}(f) \otimes_{\mathbb{R}} \mathbb{C}$ over $X$.

Proof Since $f$ is homotopic to the isometry in its polar decomposition, we may assume that $f$ itself is isometric.

The Kasparov product of $\tilde{\Theta}_{E}^{X}$ and $f^{!}$is easy to compute because the latter is a *-homomorphism. We get $\mathscr{P}_{E}^{X}$ with the usual action of $G$ and of $\mathscr{C}_{0}(X)$ by pointwise multiplication-because $\pi \circ f=\pi: E \rightarrow X$. The multiplier $F_{E}^{X}$ above is changed, however, to

$$
(x, \xi) \mapsto f(\xi) \cdot\left(1+\|f(\xi)\|^{2}\right)^{-1 / 2}=f(\xi) \cdot\left(1+\|\xi\|^{2}\right)^{-1 / 2} .
$$


The construction of $\tilde{\Theta}_{E}^{X}$ uses the left regular representation $\operatorname{Cliff}(E) \rightarrow \operatorname{End}(S)$, where $S=\operatorname{Cliff}(E)$, of the Clifford algebra on itself. The left regular representation can be extended to an irreducible representation

$$
c: \operatorname{Cliff}\left(E \oplus E^{-}\right) \cong \operatorname{Cliff}(E) \hat{\otimes} \operatorname{Cliff}(E) \rightarrow \operatorname{End}(S)
$$

of $\operatorname{Cliff}\left(E \oplus E^{-}\right)$, where $E^{-}$denotes $E$ with bilinear form negated. That is, $S$ has the structure of a spinor bundle over $\operatorname{Cliff}\left(E \oplus E^{-}\right)$. To see this, we combine the right regular representation of $E$, which commutes with the left regular one, with the grading $\gamma$; since $E$ is contained in the odd part of $\operatorname{Cliff}(E)$, the two maps

$$
\operatorname{Cliff}(E) \rightrightarrows \operatorname{Cliff}(E), \quad a \mapsto x \cdot a, \quad a \mapsto \gamma a \gamma \cdot y
$$

for $x, y \in E$ anti-commute, and the square of the latter maps $a$ to $-a \cdot y^{2}=-a\|y\|^{2}$. The fact that we get an irreducible representation follows by counting dimensions.

We use the spinor bundle $S=\operatorname{Cliff}(E)$ for $E \oplus E^{-}$just constructed to compute the line bundle $\operatorname{sign}(f)$. The point of $\operatorname{sign}(f)$ is that we have a canonical equivariant isomorphism of complex vector bundles

$$
\operatorname{sign}(A) \otimes_{\mathbb{R}}(S, c) \cong\left(S, c^{\prime}\right), \quad \varphi \otimes a \mapsto \varphi(a),
$$

where $c^{\prime}$ as usual denotes the Clifford multiplication twisted by $f \oplus$ id. This isomorphism is equivariant with respect to the ordinary (untwisted) Clifford multiplication $(x, y) \mapsto c(x, y) \otimes$ id on its domain, and the twisted action $(x, y) \mapsto c^{\prime}(x, y)=$ $c(f(x), y)$ on its co-domain. It is easy to check that the isomorphism (17) respects ordinary right Clifford multiplication (because the grading $\gamma$ can be built out of left Clifford multiplication) whence we have a $G$-equivariant isomorphism of right $\mathscr{C}_{0}(X, \operatorname{Cliff}(E))$-modules respecting left Clifford multiplication. This shows that the twisted Clifford multiplication that appears in $\tilde{\Theta}_{E}^{X} \otimes_{\mathscr{P}_{E}^{X}}\left[f^{!}\right]$is isomorphic to the standard Clifford multiplication on $\operatorname{sign}(f) \otimes \operatorname{Cliff}(E)$. Thus

$$
\tilde{\Theta}_{E}^{X} \otimes_{\mathscr{P}_{E}^{X}}\left[f^{!}\right]=[\operatorname{sign}(f)] \otimes_{\mathscr{C}_{0}(X)} \tilde{\Theta}_{E}^{X} .
$$

Since $D_{E}^{X}=\left(\tilde{\Theta}_{E}^{X}\right)^{-1}$ by the untwisted Thom isomorphism, the product with $D_{E}^{X}$ yields the class of $\operatorname{sign}(f)$ in $\mathscr{R} \mathrm{KK}_{0}^{G}\left(X ; \mathscr{C}_{0}(X), \mathscr{C}_{0}(X)\right)$.

\subsection{Computing the Lefschetz invariant}

Now we compute the Lefschetz map for a smooth $G$-equivariant map $\psi: X \rightarrow X$ that satisfies the prerequisites of Theorem 2, using the Kasparov dual involving Clifford algebras described above.

Throughout this section, we let $Y:=\operatorname{Fix}(\psi)$; this is a closed submanifold of $X$ by assumption. Let $v$ be its normal bundle; this is a vector bundle over $Y$ via the projection map $\pi: v \rightarrow Y$. Since $Y$ is a closed submanifold, it has a tubular neighbourhood $V$, that is, $V \cong v$ via a $G$-equivariant diffeomorphism whose restriction to $Y$ is the zero 
section of $v$. Extending functions by 0 outside $V$, we get canonical embeddings such as

$$
j: \mathscr{C}_{0}(v) \rightarrow \mathscr{C}_{0}(X), \quad j: \mathscr{C}_{0}\left(v, \operatorname{Cliff}\left(\left.T X\right|_{v}\right)\right) \rightarrow \mathscr{C}_{0}(X, \operatorname{Cliff}(T X))=\mathscr{P}
$$

Recall that

$$
\operatorname{Lef}(\psi)=\bar{\Theta} \otimes_{\mathscr{C}_{0}(X) \otimes \mathscr{P}}\left[\mu_{\psi}\right] \otimes \mathscr{P} D
$$

Lemma 1 shows that $\mu_{\psi}: \mathscr{C}_{0}(X, \mathscr{P}) \cong \mathscr{C}_{0}(X) \otimes \mathscr{P} \rightarrow \mathscr{P}$ is given by

$$
\mu_{\psi}(\varphi)(x)=\varphi(\psi(x), x) \in \operatorname{Cliff}\left(T_{x} X\right) \quad \text { for all } \varphi \in \mathscr{C}_{0}(X, \mathscr{P}), x \in X
$$

We are going to compose this *-homomorphism with $\bar{\Theta}$ and simplify the result:

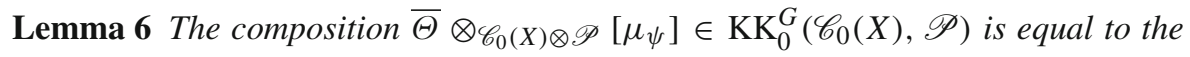
composite

$$
\begin{aligned}
\mathscr{C}_{0}(X) \stackrel{r_{Y}}{\longrightarrow} \mathscr{C}_{0}(Y) \stackrel{\operatorname{sign}\left(\mathrm{id}_{v}-D_{v} \psi\right)}{\longrightarrow} \mathscr{C}_{0}(Y) \stackrel{\tilde{\Theta}_{v}^{Y}}{\longrightarrow} \mathscr{C}_{0}(v, \operatorname{Cliff}(v)) \\
\stackrel{i}{\rightarrow} \mathscr{C}_{0}\left(v, \operatorname{Cliff}\left(\left.T X\right|_{\nu}\right)\right) \stackrel{j}{\rightarrow} \mathscr{C}_{0}(X, \operatorname{Cliff}(T X))=\mathscr{P}
\end{aligned}
$$

Here $r_{Y}$ is the restriction map; $\operatorname{sign}\left(\mathrm{id}_{v}-D_{\nu} \psi\right) \in \mathrm{KK}_{0}^{G}\left(\mathscr{C}_{0}(Y), \mathscr{C}_{0}(Y)\right)$ is the class associated to the corresponding line bundle; $\tilde{\Theta}_{v}^{Y}$ is as in Sect. 5.2; the map i is induced by the embedding

$$
\operatorname{Cliff}(v) \rightarrow \operatorname{Cliff}(v) \otimes \operatorname{Cliff}(T Y) \cong \operatorname{Cliff}(v \oplus T Y) \cong \operatorname{Cliff}\left(\left.T X\right|_{v}\right)
$$

where the first map uses the unit element in $\operatorname{Cliff}(T Y)$; and $j$ is induced by the embedding of $v$ in $X$.

Proof We have described $\Theta$ and hence $\bar{\Theta}$ by an explicit cycle. To get

$$
\bar{\Theta} \otimes \mathscr{C}_{0}(X, \mathscr{P})\left[\mu_{\psi}\right] \in \mathrm{KK}_{0}^{G}\left(\mathscr{C}_{0}(X), \mathscr{P}\right),
$$

we restrict this cycle to the graph of $\psi$. Recall that $\Theta$ is supported in a certain $G$-invariant open neighbourhood $U$ of the diagonal of $X$ in $X \times X$. We let

$$
U^{\prime}:=\{x \in X \mid(x, \psi x) \in U\}
$$

this is a $G$-invariant neighbourhood of the fixed point submanifold $Y:=\operatorname{Fix}(\psi)$. Restriction to the graph of $\psi$ replaces $J_{U}$ by the ideal

$$
J_{U}^{\prime}:=\left\{f \in \mathscr{C}_{0}(X, \operatorname{Cliff}(T X)) \mid f(x)=0 \text { unless }(x, \psi(x)) \in U\right\}
$$


in $\mathscr{P}$. The group $G$ acts on $J_{U}^{\prime}$ in the obvious way, and $\mathscr{C}_{0}(X)$ acts on $J_{U}^{\prime}$ by pointwise multiplication: $(f \cdot \varphi)(x):=f(x) \cdot \varphi(x)$. The multiplier $F$ described in Sect. 5.1 yields the multiplier

$$
F^{\prime}(x):=F(x, \psi x) \in T_{x} X \subseteq \operatorname{Cliff}\left(T_{x} X\right)
$$

where $F(x, \psi x)$ is the pre-image of $\psi(x)$ under a suitably rescaled exponential map at $x$.

In the construction of the Kasparov dual for $X$, we may choose $U$ to be an arbitrarily small neighbourhood of the diagonal. For a suitable choice of $U$, the neighbourhood $U^{\prime}$ of $Y$ will be contained in $V$, a tubular neighbourhood around $Y$. We assume this from now on.

Let $\pi: V \cong v \rightarrow Y$ be the retraction from the Tubular Neighbourhood Theorem. Since this is a $G$-equivariant deformation retraction, pointwise multiplication by $f(x)$ and $f \circ \pi(x)$ is $G$-equivariantly homotopic. Therefore, we may replace the action of $\mathscr{C}_{0}(X)$ on $J_{U}^{\prime}$ by the one of pointwise multiplication with $f \circ \pi$ for $f \in \mathscr{C}_{0}(X)$. This factors $\bar{\Theta} \otimes_{\mathscr{C}_{0}(X, \mathscr{P})}\left[\mu_{\psi}\right]$ through the restriction map $\mathscr{C}_{0}(X) \rightarrow \mathscr{C}_{0}(Y)$.

Equip $v$ with some Euclidean inner product and transport the resulting norm to $V$ via the diffeomorphism $V \cong v$. Since $U$ is a neighbourhood of the diagonal, $U^{\prime}$ is a neighbourhood of $Y$ in $X$. Let $\varrho: Y \rightarrow \mathbb{R}_{>0}$ be a $G$-invariant function with $x \in U^{\prime}$ for all $x \in V$ with $\|x\| \leq \varrho \circ \pi(x)$. Since $F(x, \psi(x))$ does not vanish unless $x \in Y$, we can rescale $F^{\prime}$ such that $F^{\prime}(x)^{2}=1$ for all $x \in U^{\prime}$ with $\|x\| \geq \varrho \circ \pi(y)$. This yields a homotopic cycle. Now we may restrict $J_{U}^{\prime}$ to the smaller ideal of elements of $\mathscr{P}$ supported in

$$
U^{\prime \prime}:=\{x \in V \mid\|x\|<\varrho \circ \pi(x)\}
$$

because the operator $F^{\prime}$ has become unitary on the complement, resulting in our Kasparov cycle being degenerate there. This neighbourhood is another tubular neighbourhood of $Y$ by a rescaling. Changing our tubular neighbourhood, we can therefore achieve that $\varrho$ becomes the constant function 1 to simplify.

We define another function

$$
\tilde{F}: V \cong v \rightarrow T X, \quad \tilde{F}(y, \xi):=(\mathrm{id}-D \psi)(\xi)
$$

for $y \in Y, \xi \in v_{y}$; here we use some $G$-equivariant section for the quotient map $T X \rightarrow$ $\nu$. We join $\tilde{F}$ and $F^{\prime}$ by the linear homotopy $t F^{\prime}+(1-t) \tilde{F}$. Since $\psi(x) \approx x+D \psi(x)$ for $x$ near $Y$, there is a neighbourhood $\tilde{V}$ of $Y$ such that $t F^{\prime}+(1-t) \tilde{F}$ is invertible on $\tilde{V} \backslash Y$. Rescaling $\tilde{F}$ and $F^{\prime}$ first, so that they become unitary outside $\tilde{V}$, and also rescaling the above homotopy, we connect our Kasparov cycle to $\left(\left.\mathscr{P}\right|_{\tilde{V}}, \tilde{F}\right)$, with $\mathscr{C}_{0}(X)$ acting by pointwise multiplication combined with $\pi:\left(f_{1} \cdot f_{2}\right)(x)=f_{1} \circ \pi(x) \cdot f_{2}(x)$.

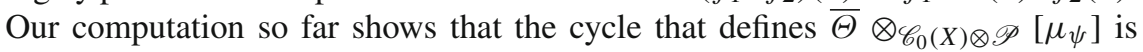
homotopic to $r_{Y} \otimes_{\mathscr{C}_{0}(Y)} \tilde{\Theta}_{v}^{Y} \otimes_{\mathscr{C}_{0}(v, \operatorname{Cliff}(v))}\left[\left(\operatorname{id}_{v}-D_{v} \psi\right)^{!}\right] \otimes_{\mathscr{C}_{0}(v, \operatorname{Cliff}(v))}(j \circ i)-$ the changes in our choice of the tubular neighbourhood above do not matter. Finally, 
Proposition 1 yields

$$
\tilde{\Theta}_{v}^{Y} \otimes_{\mathscr{C}_{0}(v, \operatorname{Cliff}(v))}\left[\left(\mathrm{id}_{v}-D_{\nu} \psi\right)^{!}\right]=\operatorname{sign}\left(\mathrm{id}_{v}-D_{v} \psi\right) \otimes_{\mathscr{C}_{0}(Y)} \tilde{\Theta}_{v}^{Y}
$$

because $\tilde{\Theta}_{v}^{Y}$ and $D_{v}^{Y}$ are inverse to each other.

To get Lef $(\psi)$, we must compose the product computed in Lemma 6 with $D \in$ $\mathrm{KK}_{0}^{G}(\mathscr{P}, \mathbb{1})$. To begin with, we compose $D$ with the class of the *-homomorphism $j \circ i: \mathscr{C}_{0}(v, \mathrm{Cliff}(v)) \rightarrow \mathscr{P}$. This yields the class of the operator $\mathrm{D}_{\mathrm{dR}}$ on the space of sections $L^{2}\left(\Lambda_{\mathbb{C}}^{*}\left(T^{*} X\right)\right)$ over $X$ of the bundle $\Lambda_{\mathbb{C}}^{*}\left(T^{*} X\right)$, with $\mathscr{C}_{0}(\nu, \mathrm{Cliff}(v))$ acting by left multiplication; here we extend such functions by 0 outside $v \cong V$ and use the embedding $\operatorname{Cliff}(v) \rightarrow \operatorname{Cliff}\left(\left.T X\right|_{\nu}\right)$. This Kasparov cycle is highly degenerate: we may restrict to the subspace of differential forms in $L^{2}\left(\Lambda_{\mathbb{C}}^{*}\left(T^{*} X\right)\right)$ that vanish outside $v$; the result is isomorphic to the bundle of forms on $v$ with respect to a complete Riemannian metric on $v$. The restriction of $\mathrm{D}_{\mathrm{dR}}$ to differential forms on $X$ which are supported in $v$ is homotopic to the Euler operator for $v$ because both operators are pseudodifferential and have the same principal symbol, up to the isomorphism involved in changing the metric. Thus we now want to compose the Euler operator on $v$ with $\tilde{\Theta}_{Y}^{v}$.

We may split the class of the Euler operator on $v$ as a Kasparov product of the class $D_{\nu}^{Y} \in \mathrm{KK}_{0}^{G}\left(\mathscr{C}_{0}(v, \operatorname{Cliff}(v)), \mathscr{C}_{0}(Y)\right)$ and the class in $\mathrm{KK}_{0}^{G}\left(\mathscr{C}_{0}(Y), \mathbb{1}\right)$ of the Euler operator for $Y$ :

$$
(j i)^{*}(D)=D_{v}^{Y} \otimes_{\mathscr{C}_{0}(Y)} \operatorname{Eul}_{Y}
$$

This is proved like the corresponding assertion for Dirac operators and amounts again to the corresponding fact about the symbols. Finally, the class $D_{v}^{Y}$ cancels $\tilde{\Theta}_{v}^{Y}$ in Lemma 6 and yields $\operatorname{Lef}(\psi)=r_{Y} \otimes_{\mathscr{C}_{0}(Y)}\left[\operatorname{sign}\left(\operatorname{id}_{v}-D_{v} \psi\right)\right] \otimes_{\mathscr{C}_{0}(Y)} \operatorname{Eul}_{Y}$ as asserted. This finishes the proof of Theorem 2.

\section{Conclusion and outlook}

We have used duality in bivariant KK-theory to refine the Lefschetz number of a selfmap to an equivariant K-homology class, and we have computed this invariant for suitable self-maps of simplicial complexes and smooth manifolds. In both cases, the Lefschetz invariant only sees a small neighbourhood of the fixed point subset.

In the simplicial case, the equivariant Lefschetz invariant is a 0 -dimensional object in the sense that it is a difference of two equivariant *-homomorphisms to a $C^{*}$-algebra of compact operators. This is a special feature of Lefschetz invariants of self-maps. Therefore, it is interesting to extend the computation of the Lefschetz invariant to more general classes in $\mathrm{KK}_{*}^{G}\left(\mathscr{C}_{0}(X), \mathscr{C}_{0}(X)\right)$ or even $\mathrm{RKK}_{*}^{G}\left(X ; \mathscr{C}_{0}(X), \mathbb{C}\right)$. Since the Lefschetz map is a split surjection on the latter group, this will necessarily lead to more complicated K-homology classes.

A geometric computation of the Lefschetz map in this case requires descriptions of the relevant Kasparov groups in terms of geometric cycles, and the use of a dual that 
is appropriate to this situation. This geometric computation of the Lefschetz invariant will be the subject of a forthcoming article.

Acknowledgments This research was supported by the National Science and Engineering Research Council of Canada Discovery Grant program, the Marie Curie Action Noncommutative Geometry and Quantum Groups (Contract MKTD-CT-2004-509794), and by the German Research Foundation [Deutsche Forschungsgemeinschaft (DFG)] through the Institutional Strategy of the University of Göttingen.

Open Access This article is distributed under the terms of the Creative Commons Attribution Noncommercial License which permits any noncommercial use, distribution, and reproduction in any medium, provided the original author(s) and source are credited.

\section{References}

1. Baum, P., Block, J.: Equivariant bicycles on singular spaces. C. R. Acad. Sci. Paris Sér. I Math. 311(2), 115-120 (1990) (English, with French summary). MR 1065441

2. Baum, P., Douglas, R.G.: $K$-homology and index theory. Operator algebras and applications, Part I (Kingston, Ont., 1980). In: Proc. Sympos. Pure Math., vol. 38, pp. 117-173. American Mathematical Society, Providence (1982). MR 679698

3. Connes, A., Skandalis, G.: The longitudinal index theorem for foliations. Publ. Res. Inst. Math. Sci., 20(6), 1139-1183 (1984). MR 775126

4. Echterhoff, S., Emerson, H., Kim, H.-J.: Fixed point formulas for proper actions (2007, to appear). arXiv: 0708.4279

5. Emerson, H., Meyer, R.: Euler characteristics and Gysin sequences for group actions on boundaries. Math. Ann. 334(4), 853-904 (2006). MR 2209260

6. Emerson, H., Meyer, R.: Dualities in equivariant Kasparov theory (2007). eprint. arXiv: 0711.0025

7. Illman, S.: Existence and uniqueness of equivariant triangulations of smooth proper $G$-manifolds with some applications to equivariant Whitehead torsion. J. Reine Angew. Math. 524 (2000), 129-183. MR 1770606

8. Kasparov, G.G.: Equivariant $K K$-theory and the Novikov conjecture. Invent. Math. 91(1), 147-201 (1988). MR 918241

9. Kasparov, G.G., Skandalis, G.: Groups acting on buildings, operator $K$-theory, and Novikov's conjecture, $K$-Theory, 4(4), 303-337 (1991). MR 1115824

10. Lück, W., Rosenberg, J.: The equivariant Lefschetz fixed point theorem for proper cocompact $G$-manifolds. High-dimensional manifold topology, pp. 322-361. World Scientific Publication, River Edge (2003). MR 2048727

11. Le Gall, P.-Y.: Théorie de Kasparov équivariante et groupoïdes. I. K-Theory, 16(4), 361-390 (1999) (French, with English and French summaries). MR 1686846

12. Weber, J.: The universal functorial equivariant Lefschetz invariant. $K$-Theory, 36(1-2), 169-207 (2006). MR 2274162 